\title{
The Bogomolov multiplier of Lie algebras
}

\author{
Z. Araghi $\operatorname{Rostami}^{1}$ (D), M. Parvizi*1 (D), P. Niroomand ${ }^{1,2}$ (it) \\ ${ }^{1}$ Department of Pure Mathematics Ferdowsi University of Mashhad, Mashhad, Iran \\ ${ }^{2}$ School of Mathematics and Computer Science Damghan University, Damghan, Iran
}

\begin{abstract}
In this paper, we extend the notion of the Bogomolov multipliers and the CP-extensions to Lie algebras. Then, we compute the Bogomolov multipliers for Abelian, Heisenberg and nilpotent Lie algebras of class at most 6. Finally, we compute the Bogomolov multipliers of complex simple and semisimple Lie algebras.
\end{abstract}

Mathematics Subject Classification (2010). 14E08, 19C09, 17C30

Keywords. commutativity-preserving exterior product, $\tilde{B}_{0}$-pairing, curly exterior product, Bogomolov multiplier, Heisenberg algebra

\section{Introduction}

During the study of continuous transformation groups in the end of 19th century, Sophus Lie found Lie algebras as a new algebraic structure. This new structure played an important role in 19 th and 20th centuries mathematical physics. (See [20,28], for more information). Lie theory is studying objects like Lie algebras, Lie groups, Root systems, Weyl groups, Linear algebraic groups, etc. and some researches show its emphasis on modern mathematics. (See $[5,20]$ for more information). Furthermore, it is shown that one can associate a Lie algebra to a continuous or Lie group. For example, Lazard introduced a correspondence between some groups and some Lie algebras. (See [19], for more information). So theories of groups and Lie algebras are structurally similar and many concepts related to groups are defined analogously to Lie algebras. In this paper we want to define the Bogomolov multipliers for Lie algebras. This concept is known for groups and it is a group-theoretical invariant introduced as an obstruction to a problem in algebraic geometry which is called the rationality problem. This problem can be stated in the following way. Let $V$ be a faithful representation of a group $G$ over a field $K$. Then $G$ acts naturally on the field of rational functions $K(V)$. Now the rationality problem or Noether's problem) can be stated as "is the field of $G$-invariant functions $K(V)^{G}$ is rational (purely transcendental) over $K$ ?" A question related to the above mentioned is whether there exist independent variables $x_{1}, \ldots, x_{r}$ such that $K(V)^{G}\left(x_{1}, \ldots, x_{r}\right)$ becomes a pure transcendental extension of $K$ ? Saltman in [25] give some examples of groups of order $p^{9}$ for which the answer to the Noether's problem was negative, even when taking $K=\mathbb{C}$.

*Corresponding Author.

Email addresses: araghirostami@gmail.com, zeinabaraghirostami@stu.um.ac.ir (Z.A. Rostami), parvizi@ferdowsi.um.ac.ir (M. Parvizi), niroomand@du.ac.ir, p_niroomand@yahoo.com (P. Niroomand)

Received: 27.08.2018; Accepted: 27.08.2019 
He used the notion of the unramified cohomology group $H_{n r}^{2}\left(\mathbb{C}(V)^{G}, \mathbb{Q} / \mathbb{Z}\right)$. Bogomolov in [4] proved that it is canonically isomorphic to

$$
B_{0}(G)=\bigcap \operatorname{ker}\left\{\operatorname{res}_{G}^{A}: H^{2}(G, \mathbb{Q} / \mathbb{Z}) \rightarrow H^{2}(A, \mathbb{Q} / \mathbb{Z})\right\},
$$

where $A$ is an abelian subgroup of $G$. The group $B_{0}(G)$ is a subgroup of the Schur multiplier $\mathcal{M}(G)=H^{2}(G, \mathbb{Q} / \mathbb{Z})$ and Kunyavskii in [18] named it the Bogomolov multiplier of $G$. Thus non triviallity of the Bogomolov multiplier leads to counter-examples to Noether's problem. But it's not always easy to calculate Bogomolov multipliers of groups. Moravec in [22] introduced an equivalent definition of the Bogomolov multiplier. In this sense, he used a notion of the non abelian exterior square $G \wedge G$ of a group $G$ to obtain a new description of the Bogomolov multiplier. He showed that if $G$ is a finite group, then $B_{0}(G)$ is non-canonically isomorphic to $\operatorname{Hom}\left(\tilde{B}_{0}(G), \mathbb{Q} / \mathbb{Z}\right)$, where the group $\tilde{B}_{0}(G)$ can be described as a section of the non abelian exterior square of the group $G$. Also, he proved that $\tilde{B}_{0}(G) \cong \mathcal{M}(G) / \mathcal{M}_{0}(G)$, such that the Schur multiplier $\mathcal{N}(G)$ or the same $H^{2}(G, \mathbb{Q} / \mathbb{Z})$ interpreted as the kernel of the commutator homomorphism $G \wedge G \rightarrow[G, G]$ given by $x \wedge y \rightarrow[x, y]$, and $\mathcal{M}_{0}(G)$ is the subgroup of $\mathcal{M}(G)$ defined as $\mathcal{M}_{0}(G)=<x \wedge y \mid[x, y]=$ $0, x, y \in G>$. Thus in the class of finite groups, $\tilde{B}_{0}(G)$ is non-canonically isomorphic to $B_{0}(G)$. With this definition and similar to the Schur multiplier, the Bogomolov multiplier can be explained as a measure of the extent to which relations among commutators in a group fail to be consequences of universal relation. Furthermore, Moravec's method relates the Bogomolov multiplier to the concept of commuting probability of a group and shows that the Bogomolov multiplier plays an important role in commutativity preserving central extensions of groups, that are famous cases in K-theory. Now, It is interesting that the analogous theory of commutativity preserving exterior product can be developed to the field of Lie theory. In this paper, we introduce a non abelian commutativity preserving exterior product, and the Bogomolov multiplier of Lie algebras. Then we investigate their properties. Moreover we compute the Bogomolov multiplier for Heisenberg Lie algebras, nilpotent Lie algebras of dimension at most 6 and complex simple and semisimple Lie algebras.

\section{Some notations and preliminaries}

Let $L$ be a finite dimensional Lie algebra. The following standard notations will be used throughout the paper.

- $[.,$.$] the Lie bracket.$

- $L^{2}=[L, L]$ the commutator subalgebra of $L$.

- $H(m)$ the Heisenberg Lie algebra of dimension $2 m+1$.

- $A(n)$ the abelian Lie algebra of dimension $n$.

- $\mathcal{M}(L) \cong \frac{R \cap F^{2}}{[R, F]} \quad$ the Schur multiplier of $L$, such that $L \cong \frac{F}{R}$.

\subsection{Exterior product [8]}

Let $L$ be a Lie algebra and $M$ and $N$ be ideals of $L$. The exterior product $M \wedge N$ is a Lie algebra generated by all symbols $m \wedge n$, subject to the following relations

(i) $\lambda(m \wedge n)=\lambda m \wedge n=m \wedge \lambda n$,

(ii) $\left(m+m^{\prime}\right) \wedge n=m \wedge n+m^{\prime} \wedge n$,

(iii) $m \wedge\left(n+n^{\prime}\right)=m \wedge n+m \wedge n^{\prime}$,

(iv) $\left[m, m^{\prime}\right] \wedge n=m \wedge\left[m^{\prime}, n\right]-m^{\prime} \wedge[m, n]$,

(v) $m \wedge\left[n, n^{\prime}\right]=\left[n^{\prime}, m\right] \wedge n-[n, m] \wedge n^{\prime}$,

(vi) $\left[(m \wedge n),\left(m^{\prime} \wedge n^{\prime}\right)\right]=-[n, m] \wedge\left[m^{\prime}, n^{\prime}\right]$,

(vii) If $m=n$, then $m \wedge n=0$, 
for all $\lambda \in F, m, m^{\prime} \in M$ and $n, n^{\prime} \in N$.

\subsection{Exterior pairing [8]}

Let L be a Lie algebra. A function $\phi: M \times N \rightarrow L$ is called an exterior pairing, if we have

(i) $h(\lambda m, n)=h(m, \lambda n)=\lambda h(m, n)$,

(ii) $h\left(m+m^{\prime}, n\right)=h(m, n)+h\left(m^{\prime}, n\right)$,

(iii) $h\left(m, n+n^{\prime}\right)=h(m, n)+h\left(m, n^{\prime}\right)$,

(iv) $h\left(\left[m, m^{\prime}\right], n\right)=h\left(m,\left[m^{\prime}, n\right]\right)+h\left(m^{\prime},[n, m]\right)$,

(v) $h\left(m,\left[n, n^{\prime}\right]\right)=h\left(\left[n^{\prime}, m\right], n\right)+h\left([m, n], n^{\prime}\right)$,

(vi) $\left[h(m, n), h\left(m^{\prime}, n^{\prime}\right)\right]=h\left([m, n],\left[m^{\prime}, n^{\prime}\right]\right)$,

(vii) If $m=n$, then $h(m, n)=0$,

for all $\lambda \in F, m, m^{\prime} \in M$ and $n, n^{\prime} \in N$.

Note that the function $M \times N \rightarrow M \wedge N$ given by $(m, n) \rightarrow m \wedge n$ is the universal exterior pairing.

\section{The commutativity preserving non abelian exterior product of Lie algebras}

In this section, we intend to extend the results of $[4,6,13,14,16,18,22]$ to the theory of Lie algebras.

Definition 3.1. Let $K$ be a Lie algebra and $M$ and $N$ be ideals of $K$. A bilinear function $h: M \times N \rightarrow K$, is called a Lie- $\tilde{B}_{0}$-pairing, if we have

(i) $h(\lambda m, n)=h(m, \lambda n)=\lambda h(m, n)$,

(ii) $h\left(m+m^{\prime}, n\right)=h(m, n)+h\left(m^{\prime}, n\right)$,

(iii) $h\left(m, n+n^{\prime}\right)=h(m, n)+h\left(m, n^{\prime}\right)$,

(iv) $h\left(\left[m, m^{\prime}\right], n\right)=h\left(m,\left[m^{\prime}, n\right]\right)-h\left(m^{\prime},[m, n]\right)$,

(v) $h\left(m,\left[n, n^{\prime}\right]\right)=h\left(\left[n^{\prime}, m\right], n\right)-h\left([n, m], n^{\prime}\right)$,

(vi) $h\left([n, m],\left[m^{\prime}, n^{\prime}\right]\right)=-\left[h(m, n), h\left(m^{\prime}, n^{\prime}\right)\right]$,

(vii) If $\left[m, n^{\prime}\right]=0$, then $h\left(m, n^{\prime}\right)=0$,

for all $\lambda \in F, m, m^{\prime} \in M$ and $n, n^{\prime} \in N$.

Definition 3.2. A Lie algebra Homomorphism is a linear map $H \in \operatorname{Hom}(L, M)$ between Lie algebras $L$ and $M$, such that it is compatible with the Lie bracket, that is

$$
H: L \rightarrow M \quad, \quad H([x, y])=[H(x), H(y)] .
$$

For example any vector space can be made into a Lie algebra with the trivial bracket.

Definition 3.3. A Lie- $\tilde{B}_{0}$-pairing $h: M \times N \rightarrow L$ is called universal, if for any Lie- $\tilde{B}_{0^{-}}$ pairing $h^{\prime}: M \times N \rightarrow L^{\prime}$, there is a unique Lie homomorphism $\theta: L \rightarrow L^{\prime}$ such that $\theta h=h^{\prime}$.

The following definition extends the concept of CP exterior product in [22] to the theory of Lie algebras.

Definition 3.4. Let $L$ be a Lie algebra and $M$ and $N$ be ideals of $L$. The CP exterior product $M \curlywedge N$ is the Lie algebra generated by all symbols $m \curlywedge n$ subject to the following relations

(i) $\lambda(m \curlywedge n)=\lambda m \curlywedge n=m \curlywedge \lambda n$,

(ii) $\left(m+m^{\prime}\right) \curlywedge n=m \curlywedge n+m^{\prime} \curlywedge n$,

(iii) $m \curlywedge\left(n+n^{\prime}\right)=m \curlywedge n+m \curlywedge n^{\prime}$,

(iv) $\left[m, m^{\prime}\right] \curlywedge n=m \curlywedge\left[m^{\prime}, n\right]-m^{\prime} \curlywedge[m, n]$, 
(v) $m \curlywedge\left[n, n^{\prime}\right]=\left[n^{\prime}, m\right] \curlywedge n-[n, m] \curlywedge n^{\prime}$,

(vi) $\left[(m \curlywedge n),\left(m^{\prime} \curlywedge n^{\prime}\right)\right]=-[n, m] \curlywedge\left[m^{\prime}, n^{\prime}\right]$,

(vii) If $[m, n]=0$, then $m \curlywedge n=0$,

for all $\lambda \in F, m, m^{\prime} \in M$ and $n, n^{\prime} \in N$.

In the case $M=N=L$, we call $L \curlywedge L$ the curly exterior product of $L$.

Proposition 3.5. The function $h: M \times N \rightarrow M \curlywedge N$ given by $(m, n) \longmapsto m \curlywedge n$, is a universal Lie- $\tilde{B}_{0}$-pairing.

Proof. By Definitions 3.1, 3.3 and 3.4, the proof is straightforward.

Theorem 3.6. Let $L$ be a Lie algebra and $M$ and $N$ be ideals of $L$. Then we have

$$
M \curlywedge N \cong \frac{M \wedge N}{\mathcal{M}_{0}(M, N)},
$$

where $\mathcal{M}_{0}(M, N)=<m \wedge n \mid m \in M, n \in N,[m, n]=0>$.

Proof. By using Definition 2.2, the function $h: M \times N \rightarrow M \curlywedge N$ given by $(m, n) \longmapsto$ $(m \curlywedge n)$ is an exterior pairing. So it induces a homomorphism $\tilde{h}: M \wedge N \rightarrow M \curlywedge N$, given by $(m \wedge n) \longmapsto m \curlywedge n$, for all $m \in M$ and $n \in N$. Clearly $\mathcal{M}_{0}(M, N) \subseteq \operatorname{ker} \tilde{h}$, so we have the homomorphism $h^{*}:(M \wedge N) / \mathcal{M}_{0}(M, N) \rightarrow M \curlywedge N$ given by $(m \wedge n)+$ $\mathcal{M}_{0}(M, N) \longmapsto(m \curlywedge n)$. On the other hand, the map $l^{*}: M \curlywedge N \rightarrow(M \wedge N) / \mathcal{M}_{0}(M, N)$ given by $(m \curlywedge n) \longmapsto(m \wedge n)+\mathcal{M}_{0}(M, N)$ is induced by the Lie- $\tilde{B}_{0}$-pairing $l: M \times N \rightarrow$ $(M \wedge N) / \mathcal{M}_{0}(M, N)$ given by $(m, n) \longmapsto(m \wedge n)+\mathcal{M}_{0}(M, N)$. Now it is easy to see that $h^{*} l^{*}=l^{*} h^{*}=1$. Thus $l^{*}$ is an isomorphism.

It is known that $\kappa: M \times N \rightarrow[M, N]$ given by $(m, n) \longmapsto[m, n]$ is an exterior pairing. So for all $m \in M$ and $n \in N$, it induces a homomorphism $\tilde{\kappa}: M \wedge N \rightarrow[M, N]$, such that $\tilde{\kappa}(m \wedge n)=[m, n]$. Moreover, the kernel of $\tilde{\kappa}$ is denoted by $\mathcal{M}(M, N)$. It can easily seen that $\mathcal{M}_{0}(M, N) \leq \mathcal{M}(M, N)$, thus there is a homomorphism $\kappa^{*}: M \wedge N / \mathcal{M}_{0}(M, N) \rightarrow[M, N]$ given by $m \wedge n+\mathcal{M}_{0}(M, N) \longmapsto[m, n]$, with $\operatorname{ker} \kappa^{*} \cong \mathcal{M}(M, N) / \mathcal{M}_{0}(M, N)$. Similar to groups, we denote $\mathcal{M}(M, N) / \mathcal{M}_{0}(M, N)$ by $\tilde{B}_{0}(M, N)$, and we call it the Bogomolov multiplier of the pair of Lie algebras $(M, N)$. Therefore, we have an exact sequence

$$
0 \rightarrow \tilde{B}_{0}(M, N) \rightarrow M \curlywedge N \rightarrow[M, N] \rightarrow 0 .
$$

In the case $M=N=L, \mathcal{M}_{0}(L, L)=<l \wedge l^{\prime} \mid l, l^{\prime} \in L,\left[l, l^{\prime}\right]=0>$ and we denote it by $\mathcal{M}_{0}(L)$.

It is known that the kernel of $\tilde{\kappa}: L \wedge L \rightarrow L^{2}$ given by $l \wedge l^{\prime} \longmapsto\left[l, l^{\prime}\right]$ is the Schur multiplier of $L$. On the other hand $\mathcal{M}_{0}(L) \leq \mathcal{M}(L)=$ ker $\tilde{\kappa}$. So there is a homomorphism $\kappa^{*}: L \wedge L / \mathcal{M}_{0}(L) \rightarrow L^{2}$ given by $l \wedge l^{\prime}+\mathcal{M}_{0}(L) \longmapsto\left[l, l^{\prime}\right]$ and ker $\kappa^{*} \cong \mathcal{M}(L) / \mathcal{M}_{0}(L)$. Similar to groups, we denote $\mathcal{M}(L) / \mathcal{M}_{0}(L)$ by $\tilde{B}_{0}(L)$, and we call it the Bogomolov multiplier of the Lie algebra $L$. So we have an exact sequence

$$
0 \rightarrow \tilde{B}_{0}(L) \rightarrow L \curlywedge L \rightarrow L^{2} \rightarrow 0 .
$$

Proposition 3.7. Let $L$ be a Lie algebra and $M, N$ and $K$ be ideals of $L$, such that $K \subseteq M \cap N$. Then there is an isomorphism

$$
M / K \curlywedge N / K \cong(M \curlywedge N) / T,
$$

where $T=<m \curlywedge n \mid m \in M, n \in N,[m, n] \in K>$.

Proof. The function $\phi: M \times N \rightarrow M / K \curlywedge N / K$ given by $(m, n) \rightarrow(m+K) \curlywedge(n+K)$ is a well-defined Lie- $\tilde{B}_{0}$-pairing. Thus there is a homomorphism $\phi^{*}: M \curlywedge N \rightarrow M / K \curlywedge N / K$ with $m \curlywedge n \longmapsto(m+K) \curlywedge(n+K)$. Clearly $T \subseteq \operatorname{ker} \phi^{*}$, so we have the homomorphism $\psi:(M \curlywedge N) / T \rightarrow M / K \curlywedge N / K$ given by $m \curlywedge(n+T) \longmapsto(m+K) \curlywedge(n+K)$. On the 
other hand, the map $\varphi^{*}: M / K \curlywedge N / K \rightarrow(M \curlywedge N) / T$ given by $(m+K) \curlywedge(n+K) \longmapsto$ $(m \curlywedge n)+T$ is induced by the Lie- $\tilde{B}_{0}$-pairing $\varphi: M / K \times N / K \rightarrow(M \curlywedge N) / T$ given by $(m+K, n+K) \longmapsto(m \curlywedge n)+T$. One can check that, $\varphi^{*} \psi=\psi \varphi^{*}=1$. Thus, $\varphi^{*}$ is an isomorphism, and the proof is complete.

Now, we give the behaviour of the CP exterior product respect to a direct sum of Lie algebras.

Proposition 3.8. Let $L_{1}$ and $L_{2}$ be ideals of a Lie algebra $L$. Then

$$
\left(L_{1} \oplus L_{2}\right) \curlywedge\left(L_{1} \oplus L_{2}\right) \cong L_{1} \curlywedge L_{1} \oplus L_{2} \curlywedge L_{2} .
$$

Proof. The result obtained by using a similar way to that of [8].

\section{Hopf-type formula for the Bogomolov multiplier of Lie algebras}

Let $L$ be a Lie algebra with a free presentation $L \cong F / R$. By the well-known Hopf formula [8], we have an isomorphism $\mathcal{M}(L) \cong\left(R \cap F^{2}\right) /[R, F]$. Here we intend to give the similar formula for $\tilde{B}_{0}(L)$.

In the following $K(F)$ denotes $\{[x, y] \mid x, y \in F\}$.

Proposition 4.1. Let $L$ be a Lie algebra with the free presentation $L \cong F / R$, then

$$
\tilde{B}_{0}(L) \cong \frac{R \cap F^{2}}{<K(F) \cap R>} .
$$

Proof. From [8], $L \wedge L \cong F^{2} /[R, F]$ and $L^{2} \cong F^{2} /\left(R \cap F^{2}\right)$. Moreover $\operatorname{ker} \tilde{\kappa}=\mathcal{M}(L) \cong$ $\left(R \cap F^{2}\right) /[R, F]$ and $\mathcal{M}_{0}(L)$ can be considered as the subalgebra of $F /[R, F]$ generated by all commutators in $F /[R, F]$ that belong to $\mathcal{M}(L)$. Thus we have the following isomorphism for $\mathcal{M}_{0}(L)$,

$$
\mathcal{M}_{0}(L) \cong<K\left(\frac{F}{[R, F]}\right) \cap \frac{R}{[R, F]}>=\frac{<K(F) \cap R>+[R, F]}{[R, F]}=\frac{<K(F) \cap R>}{[R, F]} .
$$

Therefore $\tilde{B}_{0}(L)=\mathcal{M}(L) / \mathcal{M}_{0}(L) \cong R \cap F^{2} /<K(F) \cap R>$ as required.

Proposition 4.2. Let $L$ be a Lie algebra and $M$ be an ideal of $L$. Then the sequence

$$
\tilde{B}_{0}(L) \rightarrow \tilde{B}_{0}\left(\frac{L}{M}\right) \rightarrow \frac{M}{<K(L) \cap M>} \rightarrow \frac{L}{L^{2}} \rightarrow \frac{L / M}{(L / M)^{2}} \rightarrow 0,
$$

is exact.

Proof. Suppose $0 \rightarrow R \rightarrow F \stackrel{\pi}{\rightarrow} L \rightarrow 0$ be a free presentation of $L$ and let

$T=\operatorname{ker}(F \rightarrow L / M)$. We have $M \cong T / R$. The inclusion maps $R \cap F^{2} \stackrel{f}{\rightarrow} T \cap F^{2}$,

$T \cap F^{2} \stackrel{g}{\rightarrow} T, \quad T \stackrel{h}{\rightarrow} F$ and $F \stackrel{k}{\rightarrow} F$ induce the sequence of homomorphisms

$\frac{R \cap F^{2}}{<K(F) \cap R>} \stackrel{f^{*}}{\rightarrow} \frac{T \cap F^{2}}{<K(F) \cap T>} \stackrel{g^{*}}{\rightarrow} \frac{T}{<K(F) \cap T>+R} \stackrel{h^{*}}{\rightarrow} \frac{F}{R+F^{2}} \stackrel{k^{*}}{\rightarrow}$

$\frac{F}{T+F^{2}} \rightarrow 0$. Note that $\frac{T}{<K(F) \cap T>+R} \cong \frac{M}{<K(L) \cap M>}, \frac{F}{R+F^{2}} \cong \frac{L}{L^{2}}$ and

$\frac{F}{T+F^{2}} \cong \frac{L / M}{(L / M)^{2}}$. Now by using Proposition 4.1, we have

$\tilde{B}_{0}(L) \cong \frac{R \cap F^{2}}{<K(F) \cap R>}$ and $\tilde{B}_{0}\left(\frac{L}{M}\right) \cong \frac{T \cap F^{2}}{<K(F) \cap T>}$. Moreover,

$\operatorname{im} f^{*}=\operatorname{ker} g^{*}=\frac{R \cap F^{2}}{<K(F) \cap T>}, \quad \operatorname{im} g^{*}=\operatorname{ker} h^{*}=\frac{T \cap F^{2}}{<K(F) \cap T>+R}$,

$\operatorname{im} h^{*}=\operatorname{ker} k^{*}=\frac{T}{R+F^{2}}$, and $K^{*}$ is an epimorphism. Therefore, the above sequence is exact. 
Proposition 4.3. Let $L$ be a Lie algebra with a free presentation $L \cong F / R$, and $M$ be an ideal of $L$, such that $T=\operatorname{ker}(F \rightarrow L / M)$. Then the sequence

$$
0 \rightarrow \frac{R \cap<K(F) \cap T>}{<K(F) \cap R>} \rightarrow \tilde{B}_{0}(L) \rightarrow \tilde{B}_{0}\left(\frac{L}{M}\right) \rightarrow \frac{M \cap L^{2}}{<K(L) \cap M>} \rightarrow 0,
$$

is exact.

Proof. Suppose $0 \rightarrow R \rightarrow F \stackrel{\pi}{\rightarrow} L \rightarrow 0$ be a free presentation of $L$ and let $T=\operatorname{ker}(F \rightarrow$ $L / M)$. We have $M \cong T / R$. The inclusion maps

$$
R \cap<K(F) \cap T>\stackrel{f}{\rightarrow} R \cap F^{2}, \quad R \cap F^{2} \stackrel{g}{\rightarrow} T \cap F^{2}
$$

and the map $T \cap F^{2} \stackrel{h}{\rightarrow}\left(T \cap F^{2}\right)+R$ induce the sequence of homomorphisms $0 \rightarrow \frac{R \cap<K(F) \cap T>}{<K(F) \cap R>} \stackrel{f^{*}}{\rightarrow} \frac{R \cap F^{2}}{<K(F) \cap R>} \stackrel{g^{*}}{\rightarrow} \frac{T \cap F^{2}}{<K(F) \cap T>} \stackrel{h^{*}}{\rightarrow}$ $\frac{\left(T \cap F^{2}\right)+R}{<K(F) \cap T>+R} \rightarrow 0$. It is straightforward to verify that $<K(L) \cap M>=\frac{<K(F) \cap T>+R}{R}$ and $M \cap L^{2}=\frac{T}{R} \cap \frac{F^{2}+R}{R}=\frac{\left(T \cap F^{2}\right)+R}{R}$.

Therefore we have

$$
\frac{M \cap L^{2}}{<K(L) \cap M>}=\frac{\left(\left(T \cap R^{2}\right)+R\right) / R}{(<K(F) \cap T>+R) / R} \cong \frac{\left(T \cap F^{2}\right)+R}{<K(F) \cap T>+R} .
$$

Now by using Proposition 4.1, we have

$\tilde{B}_{0}(L) \cong \frac{R \cap F^{2}}{<K(F) \cap R>}, \quad \tilde{B}_{0}\left(\frac{L}{M}\right) \cong \frac{T \cap F^{2}}{<K(F) \cap T>}, \quad$ and

$\operatorname{im} f^{*}=\operatorname{ker} g^{*}=\frac{R \cap<K(F) \cap T>}{<K(F) \cap R>}, \quad \operatorname{im} g^{*}=\operatorname{ker} h^{*}=\frac{R \cap F^{2}}{<K(F) \cap T>}$.

Moreover $h^{*}$ is an epimorphism. Thus, the above sequence is exact.

For groups, the Schur multiplier is a universal object of central extensions. Recently, parallel to the classical theory of central extensions, Jezernik and Moravec in $[13,14]$ developed a version of extension that preserve commutativity. They showed that the Bogomolov multiplier is also the universal object parametrizing such extensions for a given group. Now we want to introduce a similar notion for Lie algebras.

Definition 4.4. Let $L, M$ and $C$ be Lie algebras. An exact sequence of Lie algebras $0 \rightarrow M \stackrel{\chi}{\rightarrow} C \stackrel{\pi}{\rightarrow} L \rightarrow 0$ is called a commutativity preserving extension (CP extension) of $M$ by $L$, if commuting pairs of elements of $L$ have commuting lifts in $C$. A special type of $\mathrm{CP}$ extension with the central kernel is named a central $\mathrm{CP}$ extension.

Proposition 4.5. Let $e: 0 \rightarrow M \stackrel{\chi}{\rightarrow} C \stackrel{\pi}{\rightarrow} L \rightarrow 0$ be a central extension. Then e is a $C P$ extension if and only if $\chi(M) \cap K(C)=0$.

Proof. Suppose that $e$ is a $\mathrm{CP}$ central extension. Let $\left[c_{1}, c_{2}\right] \in \chi(M) \cap K(C)$, then there is a commuting lift $\left(c_{1}^{\prime}, c_{2}^{\prime}\right) \in C \times C$ of the commuting pair $\left(\pi\left(c_{1}\right), \pi\left(c_{2}\right)\right)$, such that $\pi\left(c_{1}^{\prime}\right)=\pi\left(c_{1}\right)$ and $\pi\left(c_{2}^{\prime}\right)=\pi\left(c_{2}\right)$. So for some $a, b \in \chi(M)$, we have $c_{1}^{\prime}=c_{1}+a, c_{2}^{\prime}=c_{2}+b$. Therefore $0=\left[c_{1}^{\prime}, c_{2}^{\prime}\right]=\left[c_{1}+a, c_{2}+b\right]=\left[c_{1}, c_{2}\right]$. Hence $\chi(M) \cap K(C)=0$. Conversely suppose that $\chi(M) \cap K(C)=0$. Choose $x, y \in L$ with $[x, y]=0$, we have $x=\pi\left(c_{1}\right)$ and $y=\pi\left(c_{2}\right)$ for some $c_{1}, c_{2} \in C$. Therefore $\pi\left(\left[c_{1}, c_{2}\right]\right)=0$. Hence $\left[c_{1}, c_{2}\right] \in \chi(M) \cap K(C)=0$, so $\left[c_{1}, c_{2}\right]=0$. Thus the central extension $e$ is a $\mathrm{CP}$ extension.

Definition 4.6. An abelian ideal $M$ of a Lie algebra $L$ is called a CP Lie subalgebra of $L$ if the extension $0 \rightarrow M \rightarrow L \rightarrow \frac{L}{M} \rightarrow 0$ is a CP extension. 
Moreover, by using Proposition 4.5 an abelian ideal $M$ of a Lie algebra $L$ is a CP Lie subalgebra of $L$ if $M \cap K(L)=0$.

Now we obtain an explicit formula for the Bogomolov multiplier of a direct product of two Lie algebras. The following lemma gives a free presentation for $L_{1} \oplus L_{2}$, in terms of the given free presentation for $L_{1}$ and $L_{2}$. It will be used in the rest.

Lemma 4.7. ([24, Lemma 2.1]) Let $L_{1}$ and $L_{2}$ be Lie algebras with free presentations $F_{1} / R_{1}$ and $F_{2} / R_{2}$, respectively. Let $F=F_{1} * F_{2}$ be the free product of $F_{1}$ and $F_{2}$. Then $0 \rightarrow R \rightarrow F \rightarrow L_{1} \oplus L_{2} \rightarrow 0$ is a free presentation for $L_{1} \oplus L_{2}$, where $R=R_{1}+R_{2}+\left[F_{2}, F_{1}\right]$.

Proposition 4.8. Let $L_{1}$ and $L_{2}$ be Lie algebras. Then

$$
\tilde{B}_{0}\left(L_{1} \oplus L_{2}\right) \cong \tilde{B}_{0}\left(L_{1}\right) \oplus \tilde{B}_{0}\left(L_{2}\right) .
$$

Proof. By using Lemma 4.7, we have

$$
\tilde{B}_{0}\left(L_{1} \oplus L_{2}\right)=\frac{\left(R_{1}+R_{2}+\left[F_{2}, F_{1}\right]\right) \cap\left(F_{1} * F_{2}\right)^{2}}{<K\left(F_{1} * F_{2}\right) \cap\left(R_{1}+R_{2}+\left[F_{2}, F_{1}\right]\right)>} .
$$

Let $F=F_{1} * F_{2}$, then the epimorphism $F \rightarrow F_{1} \times F_{2}$ induces the following epimorphism

$$
\begin{gathered}
\alpha: \frac{R \cap F^{2}}{<K(F) \cap R>} \rightarrow \frac{R_{1} \cap F_{1}^{2}}{<K\left(F_{1}\right) \cap R_{1}>} \oplus \frac{R_{2} \cap F_{2}{ }^{2}}{<K\left(F_{2}\right) \cap R_{2}>} \\
x+<K(F) \cap R>\longmapsto\left(x_{1}+<K\left(F_{1}\right) \cap R_{1}>, x_{2}+<K\left(F_{2}\right) \cap R_{2}>\right)
\end{gathered}
$$

where $x=x_{1}+x_{2}, x_{1} \in R_{1} \cap F_{1}^{2}$ and $x_{2} \in R_{2} \cap F_{2}^{2}$.

On the other hand

$$
\beta: \frac{R_{1} \cap F_{1}^{2}}{<K\left(F_{1}\right) \cap R_{1}>} \oplus \frac{R_{2} \cap F_{2}^{2}}{<K\left(F_{2}\right) \cap R_{2}>} \rightarrow \frac{R \cap F^{2}}{<K(F) \cap R>}
$$

given by

$$
\left(x_{1}+<K\left(F_{1}\right) \cap R_{1}>, x_{2}+<K\left(F_{2}\right) \cap R_{2}>\right) \longmapsto x+<K(F) \cap R>
$$

is a well-defined homomorphism. It is easy to check that $\beta$ is a right inverse to $\alpha$, so $\alpha$ is an epimorphism. Now, we show that $\alpha$ is a monomorphism. Let $x+\langle K(F) \cap R>\in \operatorname{ker} \alpha$, such that, $x=t_{1}+t_{2}$. So we have $t_{1} \in<K\left(F_{1}\right) \cap R_{1}>$ and $t_{2} \in<K\left(F_{2}\right) \cap R_{2}>$. Since $t_{1}, t_{2} \in<R \cap K(F)>$, then $x \in<K(F) \cap R>$. Thus $\alpha$ is a monomorphism.

\section{Computing the Bogomolov multiplier of Heisenberg Lie algebras}

We use the symbol $H(m)$ for the Heisenberg Lie algebra of dimension $2 m+1$. The Heisenberg Lie algebra $L$ is a Lie algebra such that $L^{2}=Z(L)$ and $\operatorname{dim} L^{2}=1$. Such Lie algebras are odd dimensional with basis $v_{1}, \ldots, v_{2 m}, v$ and the only non zero multiplications between basis elements are $\left[v_{2 i-1}, v_{2 i}\right]=-\left[v_{2 i}, v_{2 i-1}\right]=v$ for $i=1,2, \ldots, m$.

Theorem 5.1. With the above notations and assumptions $\tilde{B}_{0}(H(1))=0$.

Proof. Since $H(1) \wedge H(1)=<v_{1} \wedge v_{2}, v_{1} \wedge v, v_{2} \wedge v>$, an element $w \in \mathcal{M}(H(1)) \leq$ $H(1) \wedge H(1)$ can be written as $w=\alpha_{1}\left(v_{1} \wedge v_{2}\right)+\alpha_{2}\left(v_{1} \wedge v\right)+\alpha_{3}\left(v_{2} \wedge v\right)$, for $\alpha_{1}, \alpha_{2}, \alpha_{3} \in F$. Now, considering $\tilde{\kappa}: H(1) \wedge H(1) \rightarrow H(1)^{2}$ with ker $\tilde{\kappa}=\mathcal{M}(H(1))$, we have $\tilde{\kappa}(w)=0$, and hence $\alpha_{1}\left[v_{1}, v_{2}\right]+\alpha_{2}\left[v_{1}, v\right]+\alpha_{3}\left[v_{2}, v\right]=0$. On the other hand, $\left[v_{1}, v\right]=\left[v_{2}, v\right]=0$, $\left[\alpha_{1} v_{1}, v_{2}\right]=\alpha_{1}\left[v_{1}, v_{2}\right]=\alpha_{1} v=0$. Hence $v_{1} \wedge v, v_{2} \wedge v, \alpha_{1}\left(v_{1} \wedge v_{2}\right) \in \mathcal{M}_{0}(H(1))$. Thus $w \in \mathcal{M}_{0}(H(1))$, and so $\mathcal{M}(H(1)) \subseteq \mathcal{M}_{0}(H(1))$. Therefore $\tilde{B}_{0}(H(1))=0$. 
Theorem 5.2. $\tilde{B}_{0}(H(m))=0$, for all $m \geq 2$.

Proof. We know that

$$
H(m)=<v_{1}, v_{2}, \ldots v_{2 m}, v \mid\left[v_{2 i-1}, v_{2 i}\right]=-\left[v_{2 i}, v_{2 i-1}\right]=v, i=1 \ldots m>.
$$

We can see that

$H(m) \wedge H(m)=<v_{1} \wedge v_{2}, v_{1} \wedge v_{3}, \ldots, v_{1} \wedge v_{2 m}, v_{2} \wedge v_{3}, v_{2} \wedge v_{4}, \ldots, v_{2} \wedge v_{2 m}, \ldots$ $v_{2 m-1} \wedge v_{2 m}, v_{1} \wedge v, \ldots, v_{2 m} \wedge v>$.

Also for all $i, 1 \leq i \leq m, v_{i} \wedge v=v_{i} \wedge\left[v_{2 i-1}, v_{2 i}\right]=\left[v_{2 i}, v_{i}\right] \wedge v_{2 i-1}-\left[v_{2 i-1}, v_{i}\right] \wedge v_{2 i}=0$. Thus $H(m) \wedge H(m)=<v_{1} \wedge v_{2}, v_{1} \wedge v_{3}, \ldots, v_{1} \wedge v_{2 m}, v_{2} \wedge v_{3}, \ldots, v_{2} \wedge v_{2 m}, \ldots, v_{2 m-1} \wedge v_{2 m}>$. Now, for all $w \in \mathcal{M}(H(m)) \leq H(m) \wedge H(m)$, there exist $\alpha_{1}, \ldots \alpha_{2 m^{2}-2 m}, \beta_{1}, \ldots, \beta_{m} \in F$, such that $w=\alpha_{1}\left(v_{1} \wedge v_{3}\right)+\alpha_{2}\left(v_{1} \wedge v_{4}\right)+\ldots+\alpha_{2 m^{2}-2 m}\left(v_{2 m-2} \wedge v_{2 m}\right)+\beta_{1}\left(v_{1} \wedge v_{2}\right)$ $+\beta_{2}\left(v_{3} \wedge v_{4}\right)+\ldots+\beta_{m}\left(v_{2 m-1} \wedge v_{2 m}\right)$. Let $\tilde{\kappa}: H(m) \wedge H(m) \rightarrow H(m)^{2}$ be given by $x \wedge y \rightarrow[x, y]$. Since $\tilde{\kappa}(w)=0$, we have $\alpha_{1}\left[v_{1}, v_{3}\right]+\alpha_{2}\left[v_{1}, v_{4}\right]+\ldots+\alpha_{2 m^{2}-2 m}\left[v_{2 m-2}, v_{2 m}\right]+$ $\beta_{1}\left[v_{1}, v_{2}\right]+\beta_{2}\left[v_{3}, v_{4}\right]+\ldots+\beta_{m}\left[v_{2 m-1}, v_{2 m}\right]=0$. So, $\left(\beta_{1}+\beta_{2}+\ldots+\beta_{m}\right) v=0$. Hence, $w=\alpha_{1}\left(v_{1} \wedge v_{3}\right)+\alpha_{2}\left(v_{1} \wedge v_{4}\right)+\ldots+\alpha_{2 m^{2}-2 m}\left(v_{2 m-2} \wedge v_{2 m}\right)+\beta_{1}\left(v_{1} \wedge v_{2}-v_{3} \wedge v_{4}\right)+\beta_{2}\left(v_{3} \wedge\right.$ $\left.v_{4}-v_{5} \wedge v_{6}\right)+\ldots+\beta_{m-1}\left(v_{2 m-3} \wedge v_{2 m-2}-v_{2 m-1} \wedge v_{2 m}\right)$. On the other hand, $\left[v_{1}, v_{3}\right]=$ $\left[v_{1}, v_{4}\right]=\ldots=\left[v_{2 m-2}, v_{2 m}\right]=0$. Thus $v_{1} \wedge v_{3}, v_{1} \wedge v_{4}, \ldots v_{2 m-2} \wedge v_{2 m} \in M_{0}(H(m))$. We can see that $\left[v_{1}+v_{4}, v_{2}+v_{3}\right]=0$. So, $\left(v_{1}+v_{4}\right) \wedge\left(v_{2}+v_{3}\right) \in \mathcal{M}(H(m))$. Hence, $v_{1} \wedge v_{2}+v_{1} \wedge v_{3}+v_{4} \wedge v_{2}+v_{4} \wedge v_{3} \in \mathcal{M}_{0}(H(m))$. Thus $\left(v_{1} \wedge v_{2}\right)-\left(v_{3} \wedge v_{4}\right) \in \mathcal{M}_{0}(H(m))$. By a same way, we have

$$
\left(\left(v_{3} \wedge v_{4}\right)-\left(v_{5} \wedge v_{6}\right)\right), \ldots,\left(\left(v_{2 m-3} \wedge v_{2 m-2}\right)-\left(v_{2 m-1} \wedge v_{2 m}\right)\right) \in \mathcal{M}_{0}(H(m)) .
$$

Therefore $w \in \mathcal{M}_{0}(H(m))$ and so $\mathcal{M}(H(m)) \subseteq \mathcal{M}_{0}(H(m))$. Hence $\tilde{B}_{0}(H(m))=0$ as required.

Theorem 5.3. Let $L$ be an $n$-dimensional Lie algebra with $\operatorname{dim} L^{2}=1$. Then $\tilde{B}_{0}(L)=0$.

Proof. By Lemma 3.3 in [23], $L \cong H(m) \oplus A(n-2 m-1)$ for some $m$. Now using Theorem 5.2 and Proposition 4.8, we have

$$
\tilde{B}_{0}(L) \cong \tilde{B}_{0}(H(m) \oplus A(n-2 m-1)) \cong \tilde{B}_{0}(H(m)) \oplus \tilde{B}_{0}(A(n-2 m-1)) .
$$

Since $\tilde{B}_{0}(H(m))=\tilde{B}_{0}(A(n-2 m-1))=0$, the result follows.

\section{Computing the Bogomolov multiplier of nilpotent Lie algebras of di- mension at most 6}

This section is devoted to obtain the Bogomolov multiplier for the nilpotent Lie algebras of dimension at most 6 . We need the classification of these Lie algebras in $[7,9]$. The following results are obtained by using notations and terminology used in $[1,6,14,15]$.

Theorem 6.1. Let $L$ be a nilpotent Lie algebra of dimension at most 2 . Then $\tilde{B}_{0}(L)=0$.

Proof. Since $L$ is abelian, its Bogomolov multiplier is trivial.

From [9], there are two nilpotent Lie algebras of dimension 3, the abelian one, which we denote by $L_{3,1}$ and $L_{3,2} \cong H(1)$ with basis $v, v_{1}, v_{2}$ and the only non-zero Lie bracket $\left[v_{1}, v_{2}\right]=v$.

Theorem 6.2. Let $L$ be a nilpotent Lie algebra of dimension 3 . Then $\tilde{B}_{0}(L)=0$.

Proof. $L_{3,1}$ is abelian so $\tilde{B}_{0}\left(L_{3,1}\right)=0$. Now since $L_{3,2} \cong H(1)$, the result is obtained by using Theorem 5.1. 
From [9], there are three nilpotent Lie algebras of dimension 4 , which are isomorphic to $L_{4,1}, L_{4,2}, L_{4,3}$ and $L_{4, k} \cong L_{3, k} \oplus I, k=1,2$ (where $I$ is a 1-dimensional abelian ideal). $L_{4,3}$ has the basis $x_{1}, x_{2}, x_{3}, x_{4}$, with the only non-zero brackets $\left[x_{1}, x_{2}\right]=x_{3},\left[x_{1}, x_{3}\right]=x_{4}$.

Theorem 6.3. Let $L$ be a nilpotent Lie algebra of dimension 4 . Then $\tilde{B}_{0}(L)=0$.

Proof. Using Proposition 4.8 and Theorem 6.2, we have

$$
\tilde{B}_{0}\left(L_{4, k}\right) \cong \tilde{B}_{0}\left(L_{3, k}\right) \oplus \tilde{B}_{0}(I)=0 \text {, for } k=1,2 .
$$

Now let $L \cong L_{4,3}=<x_{1}, x_{2}, x_{3}, x_{4} \mid\left[x_{1}, x_{2}\right]=x_{3},\left[x_{1}, x_{3}\right]=x_{4}>$, we have $x_{2} \wedge x_{4}=x_{3} \wedge x_{4}=0$. So, $L_{4,3} \wedge L_{4,3}=<x_{1} \wedge x_{2}, x_{1} \wedge x_{3}, x_{1} \wedge x_{4}, x_{2} \wedge x_{3}>$.

Hence, for all $w \in \mathcal{M}\left(L_{4,3}\right) \leq L_{4,3} \wedge L_{4,3}$, there exist $\alpha_{1}, \alpha_{2}, \alpha_{3}, \alpha_{4} \in F$, such that $w=\alpha_{1}\left(x_{1} \wedge x_{2}\right)+\alpha_{2}\left(x_{1} \wedge x_{3}\right)+\alpha_{3}\left(x_{1} \wedge x_{4}\right)+\alpha_{4}\left(x_{2} \wedge x_{3}\right)$. Now, considering $\tilde{\kappa}: L_{4,3} \wedge L_{4,3} \rightarrow L_{4,3}^{2}$ given by $x \wedge y \rightarrow[x, y]$. Since $\tilde{\kappa}(w)=0$, we have $\alpha_{1}\left[x_{1}, x_{2}\right]+$ $\alpha_{2}\left[x_{1}, x_{3}\right]+\alpha_{3}\left[x_{1}, x_{4}\right]+\alpha_{4}\left[x_{2}, x_{3}\right]=0$. So $\alpha_{1} x_{3}+\alpha_{2} x_{4}=0$. On the other hand, $\left[x_{1}, x_{4}\right]=$ $\left[x_{2}, x_{3}\right]=\left[x_{2}, x_{4}\right]=\left[x_{3}, x_{4}\right]=0,\left[\alpha_{1} x_{1}, x_{2}\right]=\alpha_{1}\left[x_{1}, x_{2}\right]=\alpha_{1} x_{3}=0$ and $\left[\alpha_{2} x_{1}, x_{3}\right]=$ $\alpha_{2}\left[x_{1}, x_{3}\right]=\alpha_{2} x_{4}=0$. Hence $\left(x_{1} \wedge x_{4}\right),\left(x_{2} \wedge x_{3}\right), \alpha_{1}\left(x_{1} \wedge x_{2}\right), \alpha_{2}\left(x_{1} \wedge x_{3}\right) \in \mathcal{M}_{0}\left(L_{4,3}\right)$. So $\mathcal{M}\left(L_{4,3}\right) \subseteq \mathcal{M}_{0}\left(L_{4,3}\right)$. Thus $\tilde{B}_{0}\left(L_{4,3}\right)=0$.

From [9], the 5-dimensional Lie algebras are $L_{5, k} \cong L_{4, k} \oplus I$, for $k=1,2,3$. Where $I$ is a 1-dimensional abelian ideal and

- $L_{5,4} \cong<x_{1}, \ldots, x_{5} \mid\left[x_{1}, x_{2}\right]=\left[x_{3}, x_{4}\right]=x_{5}>$,

- $L_{5,5} \cong<x_{1}, \ldots, x_{5} \mid\left[x_{1}, x_{2}\right]=x_{3},\left[x_{1}, x_{3}\right]=\left[x_{2}, x_{4}\right]=x_{5}>$,

- $L_{5,6} \cong<x_{1}, \ldots, x_{5} \mid\left[x_{1}, x_{2}\right]=x_{3},\left[x_{1}, x_{3}\right]=x_{4},\left[x_{1}, x_{4}\right]=\left[x_{2}, x_{3}\right]=x_{5}>$,

- $L_{5,7} \cong<x_{1}, \ldots, x_{5} \mid\left[x_{1}, x_{2}\right]=x_{3},\left[x_{1}, x_{3}\right]=x_{4},\left[x_{1}, x_{4}\right]=x_{5}>$,

- $L_{5,8} \cong<x_{1}, \ldots, x_{5} \mid\left[x_{1}, x_{2}\right]=x_{4},\left[x_{1}, x_{3}\right]=x_{5}>$,

- $L_{5,9} \cong<x_{1}, \ldots, x_{5} \mid\left[x_{1}, x_{2}\right]=x_{3},\left[x_{1}, x_{3}\right]=x_{4},\left[x_{2}, x_{3}\right]=x_{5}>$.

Theorem 6.4. Let $L$ be a nilpotent Lie algebra of dimension 5 . Then $\tilde{B}_{0}(L) \neq 0$ if and only if $L \cong L_{5,6}$.

Proof. Using Theorem 6.3 and Proposition 4.8, $\tilde{B}_{0}\left(L_{5,1}\right)=\tilde{B}_{0}\left(L_{5,2}\right)=\tilde{B}_{0}\left(L_{5,3}\right)=0$. Let $L \cong L_{5,4}$, one can see that

$$
L_{5,4} \wedge L_{5,4}=<x_{1} \wedge x_{2}, x_{1} \wedge x_{3}, x_{1} \wedge x_{4}, x_{2} \wedge x_{3}, x_{2} \wedge x_{4}, x_{3} \wedge x_{4}>.
$$

Hence for all $w \in \mathcal{M}\left(L_{5,4}\right) \leq L_{5,4} \wedge L_{5,4}$, there exist $\alpha_{1}, \alpha_{2}, \ldots, \alpha_{6} \in F$, such that $w=$ $\alpha_{1}\left(x_{1} \wedge x_{2}\right)+\alpha_{2}\left(x_{1} \wedge x_{3}\right)+\alpha_{3}\left(x_{1} \wedge x_{4}\right)+\alpha_{4}\left(x_{2} \wedge x_{3}\right)+\alpha_{5}\left(x_{2} \wedge x_{4}\right)+\alpha_{6}\left(x_{3} \wedge x_{4}\right)$.

Since $\tilde{\kappa}(w)=0$, we have $\alpha_{1}\left[x_{1}, x_{2}\right]+\alpha_{2}\left[x_{1}, x_{3}\right]+\alpha_{3}\left[x_{1}, x_{4}\right]+\alpha_{4}\left[x_{2}, x_{3}\right]+\alpha_{5}\left[x_{2}, x_{4}\right]+$ $\alpha_{6}\left[x_{3}, x_{4}\right]=0$. Thus $\left(\alpha_{1}+\alpha_{6}\right) x_{5}=0$ and $\alpha_{1}+\alpha_{6}=0$. Hence $w=\alpha_{1}\left(\left(x_{1} \wedge x_{2}\right)-\left(x_{3} \wedge x_{4}\right)\right)+$ $\alpha_{2}\left(x_{1} \wedge x_{3}\right)+\alpha_{3}\left(x_{1} \wedge x_{4}\right)+\alpha_{4}\left(x_{2} \wedge x_{3}\right)+\alpha_{5}\left(x_{2} \wedge x_{4}\right)$. We can see that $\left[x_{1}+x_{4}, x_{2}+x_{3}\right]=0$, so $\left(x_{1}+x_{4}\right) \wedge\left(x_{2}+x_{3}\right) \in \mathcal{M}_{0}\left(L_{5,4}\right)$. Hence $\left(x_{1} \wedge x_{2}\right)+\left(x_{1} \wedge x_{3}\right)+\left(x_{4} \wedge x_{2}\right)+\left(x_{4} \wedge x_{3}\right) \in$ $\mathcal{M}_{0}\left(L_{5,4}\right)$, so $\left(x_{1} \wedge x_{2}\right)-\left(x_{3} \wedge x_{4}\right) \in \mathcal{M}_{0}\left(L_{5,4}\right)$. Also we know $\left(x_{1} \wedge x_{3}\right),\left(x_{1} \wedge x_{4}\right),\left(x_{2} \wedge x_{3}\right)$ and $\left(x_{2} \wedge x_{4}\right) \in \mathcal{M}_{0}\left(L_{5,4}\right)$. Therefore $w \in \mathcal{M}_{0}\left(L_{5,4}\right)$ and $\mathcal{M}\left(L_{5,4}\right) \subseteq \mathcal{M}_{0}\left(L_{5,4}\right)$. Thus $\tilde{B}_{0}\left(L_{5,4}\right)=0$.

Let $L \cong L_{5,5}$, it can be shown that

$$
L_{5,5} \wedge L_{5,5}=<x_{1} \wedge x_{2}, x_{1} \wedge x_{3}, x_{1} \wedge x_{4}, x_{2} \wedge x_{3}, x_{2} \wedge x_{4}, x_{3} \wedge x_{4}>.
$$

Hence for all $w \in \mathcal{M}\left(L_{5,5}\right) \leq L_{5,5} \wedge L_{5,5}$, there exist $\alpha_{1}, \alpha_{2}, \ldots, \alpha_{6} \in F$, such that $w=$ $\alpha_{1}\left(x_{1} \wedge x_{2}\right)+\alpha_{2}\left(x_{1} \wedge x_{3}\right)+\alpha_{3}\left(x_{1} \wedge x_{4}\right)+\alpha_{4}\left(x_{2} \wedge x_{3}\right)+\alpha_{5}\left(x_{2} \wedge x_{4}\right)+\alpha_{6}\left(x_{3} \wedge x_{4}\right)$.

Since $\tilde{\kappa}(w)=0$, we have $\alpha_{1}\left[x_{1}, x_{2}\right]+\alpha_{2}\left[x_{1}, x_{3}\right]+\alpha_{3}\left[x_{1}, x_{4}\right]+\alpha_{4}\left[x_{2}, x_{3}\right]+\alpha_{5}\left[x_{2}, x_{4}\right]+$ $\alpha_{6}\left[x_{3}, x_{4}\right]=0$. Thus $\alpha_{1} x_{3}+\left(\alpha_{2}+\alpha_{5}\right) x_{5}=0$, and $\alpha_{1}=\alpha_{2}+\alpha_{5}=0$. Hence $w=$ $\alpha_{1}\left(x_{1} \wedge x_{2}\right)+\alpha_{2}\left(\left(x_{1} \wedge x_{3}\right)-\left(x_{2} \wedge x_{4}\right)\right)+\alpha_{3}\left(x_{1} \wedge x_{4}\right)+\alpha_{4}\left(x_{2} \wedge x_{3}\right)$. On the other hand $\left[x_{1}, x_{4}\right]=\left[x_{2}, x_{3}\right]=\left[\alpha_{1} x_{1}, x_{2}\right]=\alpha_{1}\left[x_{1}, x_{2}\right]=\alpha_{1} x_{3}=0$. So $\left(x_{1} \wedge x_{4}\right),\left(x_{2} \wedge x_{3}\right), \alpha_{1}\left(x_{1} \wedge x_{2}\right) \in$ $\mathcal{M}_{0}\left(L_{5,5}\right)$. We can see that $\left[x_{1}+x_{2}+x_{3}, x_{1}+x_{2}+x_{4}\right]=0$, so $\left(x_{1}+x_{2}+x_{3}\right) \wedge\left(x_{1}+x_{2}+x_{4}\right) \in$ $\mathcal{M}_{0}\left(L_{5,5}\right)$. Hence $\left(x_{1} \wedge x_{4}\right)+\left(x_{2} \wedge x_{4}\right)+\left(x_{3} \wedge x_{1}\right)+\left(x_{3} \wedge x_{2}\right)+\left(x_{3} \wedge x_{4}\right) \in \mathcal{M}_{0}\left(L_{5,5}\right)$ and 
$\left(x_{1} \wedge x_{3}\right)-\left(x_{2} \wedge x_{4}\right) \in \mathcal{M}_{0}\left(L_{5,5}\right)$. Therefore $\mathcal{M}\left(L_{5,5}\right) \subseteq \mathcal{M}_{0}\left(L_{5,5}\right)$, and hence $\tilde{B}_{0}\left(L_{5,5}\right)=0$. Similarly, $\tilde{B}_{0}\left(L_{5,7}\right)=\tilde{B}_{0}\left(L_{5,8}\right)=\tilde{B}_{0}\left(L_{5,9}\right)=0$.

Let $L \cong L_{5,6}$, we obtain that

$$
L_{5,6} \wedge L_{5,6}=<x_{1} \wedge x_{2}, x_{1} \wedge x_{3}, x_{1} \wedge x_{4}, x_{1} \wedge x_{5}, x_{2} \wedge x_{3}, x_{2} \wedge x_{5}>.
$$

Hence for all $w \in \mathcal{M}\left(L_{5,6}\right) \leq L_{5,6} \wedge L_{5,6}$ there exist $\alpha_{1}, \alpha_{2}, \ldots, \alpha_{6} \in F$, such that $w=$ $\alpha_{1}\left(x_{1} \wedge x_{2}\right)+\alpha_{2}\left(x_{1} \wedge x_{3}\right)+\alpha_{3}\left(x_{1} \wedge x_{4}\right)+\alpha_{4}\left(x_{1} \wedge x_{5}\right)+\alpha_{5}\left(x_{2} \wedge x_{3}\right)+\alpha_{6}\left(x_{2} \wedge x_{5}\right)$.

Since $\tilde{\kappa}(w)=0$, we have $\alpha_{1}\left[x_{1}, x_{2}\right]+\alpha_{2}\left[x_{1}, x_{3}\right]+\alpha_{3}\left[x_{1}, x_{4}\right]+\alpha_{4}\left[x_{1}, x_{5}\right]+\alpha_{5}\left[x_{2}, x_{3}\right]+$ $\alpha_{6}\left[x_{2}, x_{5}\right]=0$. Thus $\alpha_{1} x_{3}+\alpha_{2} x_{4}+\left(\alpha_{3}+\alpha_{5}\right) x_{5}=0$. Therefore $\alpha_{1} x_{3}=\alpha_{2} x_{4}=\left(\alpha_{3}+\right.$ $\left.\alpha_{5}\right) x_{5}=0$. On the other hand, $\left[\alpha_{1} x_{1}, x_{2}\right]=\alpha_{1}\left[x_{1}, x_{2}\right]=\alpha_{1} x_{3}=0$ and $\left[\alpha_{2} x_{1}, x_{3}\right]=$ $\alpha_{2}\left[x_{1}, x_{3}\right]=\alpha_{2} x_{4}=0$. So $\alpha_{1}\left(x_{1} \wedge x_{2}\right), \alpha_{2}\left(x_{1} \wedge x_{3}\right),\left(x_{1} \wedge x_{5}\right),\left(x_{2} \wedge x_{5}\right) \in \mathcal{M}_{0}\left(L_{5,6}\right)$. Thus $w=\alpha_{3}\left(x_{1} \wedge x_{4}-x_{2} \wedge x_{3}\right)+\tilde{w}$, where $\tilde{w} \in \mathcal{M}_{0}\left(L_{5,6}\right)$. Let $B$ be a generating set for $\mathcal{M}_{0}\left(L_{5,6}\right)$, then $\mathcal{M}\left(L_{5,6}\right)=<B, x_{1} \wedge x_{4}-x_{2} \wedge x_{3}>$. Hence $\operatorname{dim} \tilde{B}_{0}\left(L_{5,6}\right)=1$. So $\tilde{B}_{0}\left(L_{5,6}\right) \cong A(1)$.

From [9], the 6-dimensional Lie algebras are $L_{6, k} \cong L_{5, k} \oplus I$, for $k=1, \ldots, 9$ where $I$ is a 1-dimensional abelian ideal and

- $L_{6,10} \cong<x_{1}, \ldots, x_{6} \mid\left[x_{1}, x_{2}\right]=x_{3},\left[x_{1}, x_{3}\right]=x_{6},\left[x_{4}, x_{5}\right]=x_{6}>$,

- $L_{6,11} \cong<x_{1}, \ldots, x_{6} \mid\left[x_{1}, x_{2}\right]=x_{3},\left[x_{1}, x_{3}\right]=x_{4},\left[x_{1}, x_{4}\right]=\left[x_{2}, x_{3}\right]=\left[x_{2}, x_{5}\right]$ $=x_{6}>$,

- $L_{6,12} \cong<x_{1}, \ldots, x_{6} \mid\left[x_{1}, x_{2}\right]=x_{3},\left[x_{1}, x_{3}\right]=x_{4},\left[x_{1}, x_{4}\right]=\left[x_{2}, x_{5}\right]=x_{6}>$,

- $L_{6,13} \cong<x_{1}, \ldots, x_{6} \mid\left[x_{1}, x_{2}\right]=x_{3},\left[x_{1}, x_{3}\right]=\left[x_{2}, x_{4}\right]=x_{5},\left[x_{1}, x_{5}\right]=\left[x_{3}, x_{4}\right]$ $=x_{6}>$,

- $L_{6,14} \cong<x_{1}, \ldots, x_{6} \mid\left[x_{1}, x_{2}\right]=x_{3},\left[x_{1}, x_{3}\right]=x_{4},\left[x_{1}, x_{4}\right]=\left[x_{2}, x_{3}\right]=x_{5}$, $\left[x_{2}, x_{5}\right]=x_{6},\left[x_{3}, x_{4}\right]=-x_{6}>$,

- $L_{6,15} \cong<x_{1}, \ldots, x_{6} \mid\left[x_{1}, x_{2}\right]=x_{3},\left[x_{1}, x_{3}\right]=x_{4},\left[x_{1}, x_{4}\right]=\left[x_{2}, x_{3}\right]=x_{5}$, $\left[x_{1}, x_{5}\right]=\left[x_{2}, x_{4}\right]=x_{6}>$,

- $L_{6,16} \cong<x_{1}, \ldots, x_{6} \mid\left[x_{1}, x_{2}\right]=x_{3},\left[x_{1}, x_{3}\right]=x_{4},\left[x_{1}, x_{4}\right]=x_{5},\left[x_{2}, x_{5}\right]=x_{6}$, $\left[x_{3}, x_{4}\right]=-x_{6}>$,

- $L_{6,17} \cong<x_{1}, \ldots, x_{6} \mid\left[x_{1}, x_{2}\right]=x_{3},\left[x_{1}, x_{3}\right]=x_{4},\left[x_{1}, x_{4}\right]=x_{5},\left[x_{1}, x_{5}\right]=\left[x_{2}, x_{3}\right]$ $=x_{6}>$,

- $L_{6,18} \cong<x_{1}, \ldots, x_{6} \mid\left[x_{1}, x_{2}\right]=x_{3},\left[x_{1}, x_{3}\right]=x_{4},\left[x_{1}, x_{4}\right]=x_{5},\left[x_{1}, x_{5}\right]=x_{6}>$,

- $L_{6,19}(\epsilon) \cong<x_{1}, \ldots, x_{6} \mid\left[x_{1}, x_{2}\right]=x_{4},\left[x_{1}, x_{3}\right]=x_{5},\left[x_{2}, x_{4}\right]=x_{6},\left[x_{3}, x_{5}\right]=\epsilon x_{6}>$,

- $L_{6,20} \cong<x_{1}, \ldots, x_{6} \mid\left[x_{1}, x_{2}\right]=x_{4},\left[x_{1}, x_{3}\right]=x_{5},\left[x_{1}, x_{5}\right]=\left[x_{2}, x_{4}\right]=x_{6}>$,

- $L_{6,21}(\epsilon) \cong<x_{1}, \ldots, x_{6} \mid\left[x_{1}, x_{2}\right]=x_{3},\left[x_{1}, x_{3}\right]=x_{4},\left[x_{2}, x_{3}\right]=x_{5},\left[x_{1}, x_{4}\right]=x_{6}$, $\left[x_{2}, x_{5}\right]=\epsilon x_{6}>$,

- $L_{6,22}(\epsilon) \cong<x_{1}, \ldots, x_{6} \mid\left[x_{1}, x_{2}\right]=x_{5},\left[x_{1}, x_{3}\right]=x_{6},\left[x_{2}, x_{4}\right]=\epsilon x_{6},\left[x_{3}, x_{4}\right]=x_{5}>$,

- $L_{6,23} \cong<x_{1}, \ldots, x_{6} \mid\left[x_{1}, x_{2}\right]=x_{3},\left[x_{1}, x_{3}\right]=\left[x_{2}, x_{4}\right]=x_{5},\left[x_{1}, x_{4}\right]=x_{6}>$,

- $L_{6,24}(\epsilon) \cong<x_{1}, \ldots, x_{6} \mid\left[x_{1}, x_{2}\right]=x_{3},\left[x_{1}, x_{3}\right]=\left[x_{2}, x_{4}\right]=x_{5},\left[x_{1}, x_{4}\right]=\epsilon x_{6}$, $\left[x_{2}, x_{3}\right]=x_{6}>$

- $L_{6,25} \cong<x_{1}, \ldots, x_{6} \mid\left[x_{1}, x_{2}\right]=x_{3},\left[x_{1}, x_{3}\right]=x_{5},\left[x_{1}, x_{4}\right]=x_{6}>$,

- $L_{6,26} \cong<x_{1}, \ldots, x_{6} \mid\left[x_{1}, x_{2}\right]=x_{4},\left[x_{1}, x_{3}\right]=x_{5},\left[x_{2}, x_{3}\right]=x_{6}>$.

Theorem 6.5. Let $L$ be a nilpotent Lie algebra of dimension 6 . Then $\tilde{B}_{0}(L) \neq 0$ if and only if $L$ is isomorphic to one of the Lie algebras $L_{6,6}, L_{6,13}, L_{6,14}, L_{6,15}, L_{6,22}(\epsilon)$ where $\epsilon \geq 1, L_{6,23}$ and $L_{6,24}(\epsilon)$ where $\epsilon \neq 1$.

Proof. Using Proposition 4.8 and Theorem 6.4, $\tilde{B}_{0}\left(L_{6,1}\right)=\tilde{B}_{0}\left(L_{6,2}\right)=\tilde{B}_{0}\left(L_{6,3}\right)=$ $\tilde{B}_{0}\left(L_{6,4}\right)=\tilde{B}_{0}\left(L_{6,5}\right)=\tilde{B}_{0}\left(L_{6,7}\right)=\tilde{B}_{0}\left(L_{6,8}\right)=\tilde{B}_{0}\left(L_{6,9}\right)=0$ and $\tilde{B}_{0}\left(L_{6,6}\right) \neq 0$. Also similar to the calculations in Theorems 6.3 and 6.4 , we have $\tilde{B}_{0}\left(L_{6,13}\right), \tilde{B}_{0}\left(L_{6,14}\right), \tilde{B}_{0}\left(L_{6,15}\right)$, $\tilde{B}_{0}\left(L_{6,23}\right) \neq 0$ and $\tilde{B}_{0}\left(L_{6,22}(\epsilon)\right)(\epsilon=0), \tilde{B}_{0}\left(L_{6,24}(\epsilon)\right)(\epsilon=1), \tilde{B}_{0}\left(L_{6,10}\right), \tilde{B}_{0}\left(L_{6,12}\right)$, $\tilde{B}_{0}\left(L_{6,16}\right), \tilde{B}_{0}\left(L_{6,17}\right), \tilde{B}_{0}\left(L_{6,18}\right), \tilde{B}_{0}\left(L_{6,20}\right), \tilde{B}_{0}\left(L_{6,25}\right), \tilde{B}_{0}\left(L_{6,26}\right)=0$. 
Let $L \cong L_{6,11}$, one can compute that

$L_{6,11} \wedge L_{6,11}=<x_{1} \wedge x_{2}, x_{1} \wedge x_{3}, x_{1} \wedge x_{4}, x_{1} \wedge x_{5}, x_{2} \wedge x_{3}, x_{2} \wedge x_{4}, x_{2} \wedge x_{5}, x_{3} \wedge x_{4}, x_{3} \wedge x_{5}, x_{4} \wedge x_{5}>$.

Hence for all $w \in \mathcal{M}\left(L_{6,11}\right) \leq L_{6,11} \wedge L_{6,11}$, there exist $\alpha_{1}, \alpha_{2}, \ldots, \alpha_{10} \in F$, such that $w=\alpha_{1}\left(x_{1} \wedge x_{2}\right)+\alpha_{2}\left(x_{1} \wedge x_{3}\right)+\alpha_{3}\left(x_{1} \wedge x_{4}\right)+\alpha_{4}\left(x_{1} \wedge x_{5}\right)+\alpha_{5}\left(x_{2} \wedge x_{3}\right)+\alpha_{6}\left(x_{2} \wedge\right.$ $\left.x_{4}\right)+\alpha_{7}\left(x_{2} \wedge x_{5}\right)+\alpha_{8}\left(x_{3} \wedge x_{4}\right)+\alpha_{9}\left(x_{3} \wedge x_{5}\right)+\alpha_{10}\left(x_{4} \wedge x_{5}\right)$. Since $\tilde{\kappa}(w)=0$, we have $\alpha_{1}\left[x_{1}, x_{2}\right]+\alpha_{2}\left[x_{1}, x_{3}\right]+\alpha_{3}\left[x_{1}, x_{4}\right]+\alpha_{4}\left[x_{1}, x_{5}\right]+\alpha_{5}\left[x_{2}, x_{3}\right]+\alpha_{6}\left[x_{2}, x_{4}\right]+\alpha_{7}\left[x_{2}, x_{5}\right]+$ $\alpha_{8}\left[x_{3}, x_{4}\right]+\alpha_{9}\left[x_{3}, x_{5}\right]+\alpha_{10}\left[x_{4}, x_{5}\right]=0$. Thus $\alpha_{1} x_{3}+\alpha_{2} x_{4}+\left(\alpha_{3}+\alpha_{5}+\alpha_{7}\right) x_{6}=0$, and $\alpha_{1}=\alpha_{2}=\alpha_{3}+\alpha_{5}+\alpha_{7}=0$. Hence $\alpha_{7}=-\alpha_{3}-\alpha_{5}$, so $w=\alpha_{3}\left(x_{1} \wedge x_{4}-x_{2} \wedge x_{5}\right)+\alpha_{4}\left(x_{1} \wedge\right.$ $\left.x_{5}\right)+\alpha_{5}\left(x_{2} \wedge x_{3}-x_{2} \wedge x_{5}\right)+\alpha_{6}\left(x_{2} \wedge x_{4}\right)+\alpha_{8}\left(x_{3} \wedge x_{4}\right)+\alpha_{9}\left(x_{3} \wedge x_{5}\right)+\alpha_{10}\left(x_{4} \wedge x_{5}\right)$. On the other hand $\left[x_{1}, x_{5}\right]=\left[x_{2}, x_{4}\right]=\left[x_{3}, x_{4}\right]=\left[x_{3}, x_{5}\right]=\left[x_{4}, x_{5}\right]=0$. So $\left(x_{1} \wedge x_{5}\right),\left(x_{2} \wedge x_{4}\right),\left(x_{3} \wedge\right.$ $\left.x_{4}\right),\left(x_{3} \wedge x_{5}\right)$ and $\left(x_{4} \wedge x_{5}\right) \in \mathcal{M}_{0}\left(L_{6,11}\right)$. We can see that $\left[x_{1}+x_{2}+x_{5}, x_{1}+x_{2}+x_{4}\right]=0$, so $\left(x_{1}+x_{2}+x_{5}\right) \wedge\left(x_{1}+x_{2}+x_{4}\right) \in \mathcal{M}_{0}\left(L_{6,11}\right)$. Hence $\left(x_{1} \wedge x_{4}-x_{2} \wedge x_{5}\right) \in \mathcal{M}_{0}\left(L_{6,11}\right)$. Also $\left[x_{2}+x_{5}, x_{2}+x_{3}\right]=0$, so $\left(x_{2} \wedge x_{3}-x_{2} \wedge x_{5}\right) \in \mathcal{M}_{0}\left(L_{6,11}\right)$. Therefore $\mathcal{M}\left(L_{6,11}\right) \subseteq \mathcal{M}_{0}\left(L_{6,11}\right)$, and $\tilde{B}_{0}\left(L_{6,11}\right)=0$.

Let $L \cong L_{6,19}(\epsilon)$ where $\epsilon \geq 0$, we can write

$L_{6,19}(\epsilon) \wedge L_{6,19}(\epsilon)=<x_{1} \wedge x_{2}, x_{1} \wedge x_{3}, x_{1} \wedge x_{4}, x_{1} \wedge x_{5}, x_{2} \wedge x_{3}, x_{2} \wedge x_{4}, x_{3} \wedge x_{4}, x_{3} \wedge x_{5}>$.

For all $w \in \mathcal{M}\left(L_{6,19}(\epsilon)\right) \leq L_{6,19}(\epsilon) \wedge L_{6,19}(\epsilon)$, there exist $\alpha_{1}, \alpha_{2}, \ldots, \alpha_{8} \in F$, such that $w=\alpha_{1}\left(x_{1} \wedge x_{2}\right)+\alpha_{2}\left(x_{1} \wedge x_{3}\right)+\alpha_{3}\left(x_{1} \wedge x_{4}\right)+\alpha_{4}\left(x_{1} \wedge x_{5}\right)+\alpha_{5}\left(x_{2} \wedge x_{3}\right)+\alpha_{6}\left(x_{2} \wedge x_{4}\right)+\alpha_{7}\left(x_{3} \wedge\right.$ $\left.x_{4}\right)+\alpha_{8}\left(x_{3} \wedge x_{5}\right)$. Since $\tilde{\kappa}(w)=0$, we have $\alpha_{1}\left[x_{1}, x_{2}\right]+\alpha_{2}\left[x_{1}, x_{3}\right]+\alpha_{3}\left[x_{1}, x_{4}\right]+\alpha_{4}\left[x_{1}, x_{5}\right]+$ $\alpha_{5}\left[x_{2}, x_{3}\right]+\alpha_{6}\left[x_{2}, x_{4}\right]+\alpha_{7}\left[x_{3}, x_{4}\right]+\alpha_{8}\left[x_{3}, x_{5}\right]=0$. Thus $\alpha_{1} x_{4}+\alpha_{2} x_{5}+\left(\alpha_{6}+\epsilon \alpha_{8}\right) x_{6}=0$, and $\alpha_{1}=\alpha_{2}=\alpha_{6}+\epsilon \alpha_{8}=0$. Hence $w=\alpha_{3}\left(x_{1} \wedge x_{4}\right)+\alpha_{4}\left(x_{1} \wedge x_{5}\right)+\alpha_{5}\left(x_{2} \wedge\right.$ $\left.x_{3}\right)+\alpha_{7}\left(x_{3} \wedge x_{4}\right)+\alpha_{8}\left(\left(x_{3} \wedge x_{5}\right)-\epsilon\left(x_{2} \wedge x_{4}\right)\right)$. Also, $\left[x_{1}, x_{4}\right]=\left[x_{1}, x_{5}\right]=\left[x_{2}, x_{3}\right]=$ $\left[x_{3}, x_{4}\right]=0$. So $\left(x_{1} \wedge x_{4}\right),\left(x_{1} \wedge x_{5}\right),\left(x_{2} \wedge x_{3}\right),\left(x_{3} \wedge x_{4}\right) \in \mathcal{M}_{0}\left(L_{6,19}(\epsilon)\right)$. We can see that $\left[x_{3}+\epsilon x_{2}+x_{4}, x_{3}+\epsilon x_{2}+x_{5}\right]=0$, so $\left(x_{3}+\epsilon x_{2}+x_{4}\right) \wedge\left(x_{3}+\epsilon x_{2}+x_{5}\right) \in \mathcal{M}_{0}\left(L_{6,19}(\epsilon)\right)$. Hence $\left(x_{3} \wedge x_{5}\right)+\epsilon\left(x_{2} \wedge x_{5}\right)+\left(x_{4} \wedge x_{3}\right)+\epsilon\left(x_{4} \wedge x_{2}\right)+\left(x_{4} \wedge x_{5}\right) \in \mathcal{M}_{0}\left(L_{6,19}(\epsilon)\right)$. Thus $\left(x_{3} \wedge x_{5}\right)-$ $\epsilon\left(x_{2} \wedge x_{4}\right) \in \mathcal{M}_{0}\left(L_{6,19}(\epsilon)\right)$. Therefore $\mathcal{M}\left(L_{6,19}(\epsilon)\right) \subseteq \mathcal{M}_{0}\left(L_{6,19}(\epsilon)\right)$, and $\tilde{B}_{0}\left(L_{6,19}(\epsilon)\right)=0$, where $\epsilon \geq 0$. Similarly $\tilde{B}_{0}\left(L_{6,21}(\epsilon)\right)=0$, where $\epsilon \geq 0$.

For $L \cong L_{6,22}(\epsilon)$ where $\epsilon \geq 1$, we have

$L_{6,22}(\epsilon) \wedge L_{6,22}(\epsilon)=<x_{1} \wedge x_{2}, x_{1} \wedge x_{3}, x_{1} \wedge x_{4}, x_{1} \wedge x_{6}, x_{2} \wedge x_{3}, x_{2} \wedge x_{4}, x_{3} \wedge x_{4}, x_{3} \wedge x_{5}, x_{3} \wedge$ $x_{6}, x_{4} \wedge x_{6}>$.

Now for all $w \in \mathcal{M}\left(L_{6,22}(\epsilon)\right) \leq L_{6,22}(\epsilon) \wedge L_{6,22}(\epsilon)$, there exist $\alpha_{1}, \alpha_{2}, \ldots, \alpha_{10} \in F$, such that $w=\alpha_{1}\left(x_{1} \wedge x_{2}\right)+\alpha_{2}\left(x_{1} \wedge x_{3}\right)+\alpha_{3}\left(x_{1} \wedge x_{4}\right)+\alpha_{4}\left(x_{1} \wedge x_{6}\right)+\alpha_{5}\left(x_{2} \wedge x_{3}\right)+\alpha_{6}\left(x_{2} \wedge\right.$ $\left.x_{4}\right)+\alpha_{7}\left(x_{3} \wedge x_{4}\right)+\alpha_{8}\left(x_{3} \wedge x_{5}\right)+\alpha_{9}\left(x_{3} \wedge x_{6}\right)+\alpha_{10}\left(x_{4} \wedge x_{6}\right)$. Since $\tilde{\kappa}(w)=0$, we have $\alpha_{1}\left[x_{1}, x_{2}\right]+\alpha_{2}\left[x_{1}, x_{3}\right]+\alpha_{3}\left[x_{1}, x_{4}\right]+\alpha_{4}\left[x_{1}, x_{6}\right]+\alpha_{5}\left[x_{2}, x_{3}\right]+\alpha_{6}\left[x_{2}, x_{4}\right]+\alpha_{7}\left[x_{3}, x_{4}\right]+$ $\alpha_{8}\left[x_{3}, x_{5}\right]+\alpha_{9}\left[x_{3}, x_{6}\right]+\alpha_{10}\left[x_{4}, x_{6}\right]=0$. Thus $\left(\alpha_{1}+\alpha_{7}\right) x_{5}+\left(\alpha_{2}+\epsilon \alpha_{6}\right) x_{6}=0$, so $\alpha_{7}=-\alpha_{1}$ and $\alpha_{2}=-\epsilon \alpha_{6}$. Hence $w=\alpha_{1}\left(x_{1} \wedge x_{2}-x_{3} \wedge x_{4}\right)+\alpha_{3}\left(x_{1} \wedge x_{4}\right)+\alpha_{4}\left(x_{1} \wedge x_{6}\right)+$ $\alpha_{5}\left(x_{2} \wedge x_{3}\right)+\alpha_{6}\left(x_{2} \wedge x_{4}-\epsilon\left(x_{1} \wedge x_{3}\right)\right)+\alpha_{8}\left(x_{3} \wedge x_{5}\right)+\alpha_{9}\left(x_{3} \wedge x_{6}\right)+\alpha_{10}\left(x_{4} \wedge x_{6}\right)$. On the other hand $\left[x_{1}, x_{4}\right]=\left[x_{1}, x_{6}\right]=\left[x_{2}, x_{3}\right]=\left[x_{3}, x_{5}\right]=\left[x_{3}, x_{6}\right]=\left[x_{4}, x_{6}\right]=0$. So $\left(x_{1} \wedge x_{4}\right),\left(x_{1} \wedge x_{6}\right),\left(x_{2} \wedge x_{3}\right),\left(x_{3} \wedge x_{5}\right),\left(x_{3} \wedge x_{6}\right)$ and $\left(x_{4} \wedge x_{6}\right) \in \mathcal{M}_{0}\left(L_{6,22}(\epsilon)\right)$. Thus $w=$ $\alpha_{1}\left(x_{1} \wedge x_{2}-x_{3} \wedge x_{4}\right)+\alpha_{6}\left(x_{2} \wedge x_{4}-\epsilon\left(x_{1} \wedge x_{3}\right)\right)+\tilde{w}$, where $\tilde{w} \in \mathcal{M}_{0}\left(L_{6,22}(\epsilon)\right)$. Let $B$ be a generating set for $\mathcal{M}_{0}\left(L_{6,22}(\epsilon)\right)$, then $\mathcal{M}\left(L_{6,22}(\epsilon)\right)=<B, x_{1} \wedge x_{2}-x_{3} \wedge x_{4}, x_{2} \wedge x_{4}-\epsilon\left(x_{1} \wedge x_{3}\right)>$. Hence $\operatorname{dim} \tilde{B}_{0}\left(L_{6,22}(\epsilon)\right) \geq 2$. So $\tilde{B}_{0}\left(L_{6,22}(\epsilon)\right) \neq 0$.

Let $L \cong L_{6,24}(\epsilon)$ where $\epsilon \neq 1$, we can compute

$L_{6,24}(\epsilon) \wedge L_{6,24}(\epsilon)=<x_{1} \wedge x_{2}, x_{1} \wedge x_{3}, x_{1} \wedge x_{4}, x_{2} \wedge x_{3}, x_{2} \wedge x_{4}, x_{2} \wedge x_{5}, x_{2} \wedge x_{6}, x_{3} \wedge x_{4}, x_{4} \wedge$ $x_{5}, x_{4} \wedge x_{6}>$.

So, for all $w \in \mathcal{M}\left(L_{6,24}(\epsilon)\right) \leq L_{6,24}(\epsilon) \wedge L_{6,24}(\epsilon)$, there exist $\alpha_{1}, \alpha_{2}, \ldots, \alpha_{10} \in F$, such 
that $w=\alpha_{1}\left(x_{1} \wedge x_{2}\right)+\alpha_{2}\left(x_{1} \wedge x_{3}\right)+\alpha_{3}\left(x_{1} \wedge x_{4}\right)+\alpha_{4}\left(x_{2} \wedge x_{3}\right)+\alpha_{5}\left(x_{2} \wedge x_{4}\right)+\alpha_{6}\left(x_{2} \wedge\right.$ $\left.x_{5}\right)+\alpha_{7}\left(x_{2} \wedge x_{6}\right)+\alpha_{8}\left(x_{3} \wedge x_{4}\right)+\alpha_{9}\left(x_{4} \wedge x_{5}\right)+\alpha_{10}\left(x_{4} \wedge x_{6}\right)$. Since $\tilde{\kappa}(w)=0$, we have $\alpha_{1}\left[x_{1}, x_{2}\right]+\alpha_{2}\left[x_{1}, x_{3}\right]+\alpha_{3}\left[x_{1}, x_{4}\right]+\alpha_{4}\left[x_{2}, x_{3}\right]+\alpha_{5}\left[x_{2}, x_{4}\right]+\alpha_{6}\left[x_{2}, x_{5}\right]+\alpha_{7}\left[x_{2}, x_{6}\right]+\alpha_{8}\left[x_{3}, x_{4}\right]+$ $\alpha_{9}\left[x_{4}, x_{5}\right]+\alpha_{10}\left[x_{4}, x_{6}\right]=0$. Thus $\alpha_{1} x_{3}+\left(\alpha_{2}+\alpha_{5}\right) x_{5}+\left(\alpha_{4}+\epsilon \alpha_{3}\right) x_{6}=0$, so $\alpha_{1}=0, \alpha_{5}=-\alpha_{2}$ and $\alpha_{4}=-\epsilon \alpha_{3}$. Hence $w=\alpha_{2}\left(x_{1} \wedge x_{3}-x_{2} \wedge x_{4}\right)+\alpha_{3}\left(x_{1} \wedge x_{4}-\epsilon\left(x_{2} \wedge x_{3}\right)\right)+\alpha_{6}\left(x_{2} \wedge\right.$ $\left.x_{5}\right)+\alpha_{7}\left(x_{2} \wedge x_{6}\right)+\alpha_{8}\left(x_{3} \wedge x_{4}\right)+\alpha_{9}\left(x_{4} \wedge x_{5}\right)+\alpha_{10}\left(x_{4} \wedge x_{6}\right)$. Also we have, $\left[x_{2}, x_{5}\right]=$ $\left[x_{2}, x_{6}\right]=\left[x_{3}, x_{4}\right]=\left[x_{4}, x_{5}\right]=\left[x_{4}, x_{6}\right]=0$. So $\left(x_{2} \wedge x_{5}\right),\left(x_{2} \wedge x_{6}\right),\left(x_{3} \wedge x_{4}\right),\left(x_{4} \wedge x_{5}\right)$ and $\left(x_{4} \wedge x_{6}\right) \in \mathcal{M}_{0}\left(L_{6,24}(\epsilon)\right)$. Thus $w=\alpha_{2}\left(x_{1} \wedge x_{3}-x_{2} \wedge x_{4}\right)+\alpha_{3}\left(x_{1} \wedge x_{4}-\epsilon\left(x_{2} \wedge x_{3}\right)\right)+\tilde{w}$, where $\tilde{w} \in \mathcal{M}_{0}\left(L_{6,24}(\epsilon)\right)$. Let $B$ be a generating set for $\mathcal{M}_{0}\left(L_{6,24}(\epsilon)\right)$, then $\mathcal{M}\left(L_{6,24}(\epsilon)\right)=<$ $B, x_{1} \wedge x_{3}-x_{2} \wedge x_{4}, x_{1} \wedge x_{4}-\epsilon\left(x_{2} \wedge x_{3}\right)>$. Hence $\operatorname{dim} \tilde{B}_{0}\left(L_{6,24}(\epsilon)\right) \geq 2$. So $\tilde{B}_{0}\left(L_{6,24}(\epsilon)\right) \neq 0$.

If $\epsilon=1$, we have $\alpha_{1} x_{3}+\left(\alpha_{2}+\alpha_{5}\right) x_{5}+\left(\alpha_{4}+\alpha_{3}\right) x_{6}=0$, so $\alpha_{1}=0, \alpha_{5}=-\alpha_{2}$ and $\alpha_{4}=-\alpha_{3}$. Hence $w=\alpha_{2}\left(x_{1} \wedge x_{3}-x_{2} \wedge x_{4}\right)+\alpha_{3}\left(x_{1} \wedge x_{4}-x_{2} \wedge x_{3}\right)+\alpha_{6}\left(x_{2} \wedge x_{5}\right)+\alpha_{7}\left(x_{2} \wedge\right.$ $\left.x_{6}\right)+\alpha_{8}\left(x_{3} \wedge x_{4}\right)+\alpha_{9}\left(x_{4} \wedge x_{5}\right)+\alpha_{10}\left(x_{4} \wedge x_{6}\right)$. On the other hand $\left[x_{1}+x_{2}+x_{4}, x_{1}+x_{2}+\right.$ $\left.x_{3}\right],\left[x_{1}+x_{2}+x_{3}, x_{1}+x_{2}+x_{4}\right]=0$. So $\left(x_{1} \wedge x_{3}-x_{2} \wedge x_{4}\right),\left(x_{1} \wedge x_{4}-x_{2} \wedge x_{3}\right) \in \mathcal{M}_{0}\left(L_{6,24}(\epsilon)\right)$. Therefore $\mathcal{M}\left(L_{6,24}(\epsilon)\right) \subseteq \mathcal{M}_{0}\left(L_{6,24}(\epsilon)\right)$, and $\tilde{B}_{0}\left(L_{6,24}(\epsilon)\right)=0$, where $\epsilon=1$.

One of the important results on the Schur multiplier of Lie algebras was presented by Moneyhun in [21]. He showed that for a Lie algebra $L$ of $\operatorname{dimension} n, \operatorname{dim} \mathcal{M}(L)=$ $n(n-1) / 2-t(L)$, for some $t(L) \geq 0$. His results suggest the interesting question, "can we classify Lie algebras of dimension $n$ by $t(L)$ ?" The answer to this question can be found for $t(L)=1, \ldots, 8$ in $[3,11,12,21]$. On the other hand, from [23], we have an upper bound for the dimension of the Schur multiplier of a non abelian nilpotent Lie algebra as $\operatorname{dim} \mathcal{M}(L)=n(n-1)(n-2) / 2+1-s(L)$, for some $s(L) \geq 0$. Hence by the same motivation, we have the analogous question for classification of $L$ according to the values of $s(L)$. It seems that classifying nilpotent Lie algebras by $s(L)$ helps to the classification of Lie algebras in term of $t(L)$. (See for instance [23]). Now, according to this classification, we will investigate the Bogomolov multiplier for some Lie algebras.

Theorem 6.6. Let $L$ be an $n$-dimensional nilpotent Lie algebra with $s(L)=1$. Then, $\tilde{B}_{0}(L)=0$.

Proof. Since $s(L)=1$, by Theorem 3.9 in [23], $L \cong L_{5,4}$. So $\tilde{B}_{0}(L)=0$.

Theorem 6.7. Let $L$ be a $n$-dimension nilpotent Lie algebra and $t(L) \leq 6$. Then, $\tilde{B}_{0}(L)=$ 0 .

Proof. By Theorem 3.10 in [23] and Proposition 4.8, $\tilde{B}_{0}(L)=0$.

Theorem 6.8. Let $L$ be an $n$-dimensional nilpotent Lie algebra with $s(L)=2$ and $\operatorname{dim} L^{2}=2$. Then $\tilde{B}_{0}(L)=0$.

Proof. By Theorem 4.3 in [23], $L \cong L_{4,3}$ or $L \cong L_{5,4} \oplus A(1)$. Thus $\tilde{B}_{0}(L)=0$.

\section{The Bogomolov multipliers of complex simple and semisimple Lie al- gebras}

A simple group is a group with no non-trivial proper normal subgroup. The classification of finite simple groups is a major milestone in the history of mathematics. On the other hand with the help of the Jordan-Holder Theorem, a finite group can be written as a certain combination of simple groups. Also, in contrast to the classification of finite simple groups, the classification of simple Lie groups is simplified by using the manifold structure. In particular every Lie group has an dependent Lie algebra and in this regard, some authors have also gained some results. For example, Bosshardt showed that a Lie group is simple if and only if its Lie algebra is simple. (see $[5,20,26]$ for more information). Theories of groups and Lie algebras are structurally similar, and many concepts related to 
groups, there are analogously defined concepts for Lie algebras. Eventually, this subject reduces the problem of finding simple Lie groups to classifying simple Lie algebras. In this section we obtain the Bogomolov multipliers of complex semisimple Lie algebras and complex simple Lie algebras.

Definition 7.1 ([10]). A Lie algebra $L$ is called semisimple if the only commutative ideal of $L$ is 0 .

For example a 0-dimension Lie algebra, the special linear Lie algebra, the odd-dimension special orthogonal Lie algebra, the symplectic Lie algebra and the even-dimension special orthogonal Lie algebra for $(n>1)$ are semisimple.

Definition $7.2([10])$. A Lie algebra $L$ is simple if it has no ideals other than 0 and $L$, and it is not abelian.

Theorem 7.3 ([27]). A Lie algebra $L$ is semisimple if and only if $L=\oplus_{i} L_{i}$, for simple Lie algebras $L_{i}$.

We can therefore view a semisimple Lie algebra $L$ as a direct sum of simple Lie algebras $L_{i}$, which have only 0 and $L_{i}$ for their ideals. In particular, every simple Lie algebra is also semisimple.

Batten in [2] (Example 2 of Chapter 2), showed that any semisimple Lie algebra over a field of characteristic 0 has a trivial Schur multiplier. Also we know the Bogomolov multiplier is subalgebra of the Schur multiplier, so these semisimple Lie algebras have trivial Bogomolov multipliers. Therefore all simple Lie algebras over a field of characteristic 0 have trivial Bogomolov multipliers.

In this section, considering the classification of simple complex Lie algebras Cartan [5], we will show that the Bogomolov multipliers of an arbitrary complex semisimple Lie algebra is trivial. Using our computational method as used in Theorems 5.1, 5.2, 6.3, 6.4 and 6.5. Note that this result is a special case of results of Batten [2] (the Example 2 of Chapter 2), but it is worthy to state it here because we prove it by using computations on the Bogomolov multiplier regardless of its relation with the Schur multiplier.

In the following, $E_{i j}$ denotes the matrix with 1 at the intersection of the $i$-th row and the $j$-th column and 0 everywhere else. The Lie bracket of $E_{i j}$ and $E_{k l}$ is given by $\left[E_{i j}, E_{k l}\right]=E_{i j} E_{k l}-E_{k l} E_{i j}=\delta_{j k} E_{i l}-\delta_{i l} E_{k j}$.

Theorem 7.4. Every following semisimple Lie algebra over $\mathbb{C}$, has trivial Bogomolov multiplier.

(i) $\operatorname{Sl}(n+1, \mathbb{C})$,

(ii) $S o(2 n+1, \mathbb{C})$,

(iii) $S p(n, \mathbb{C})$,

(iv) $\operatorname{So}(2 n, \mathbb{C}), n \geq 2$.

Proof. Let $L$ be a special linear Lie algebra $S l(n+1, \mathbb{C})$. From [17], $S l(n+1, \mathbb{C})$ has the basis $D_{i i+1}, E_{i j}$ such that $D_{i j}=E_{i i}-E_{j j}$. So for $j \neq i=1 \ldots n$, we have

$S l(n+1, \mathbb{C})=<D_{i i+1}, E_{i j} \mid\left[D_{i i+1}, D_{i+1 i+2}\right]=D_{i i+2},\left[D_{i i+1}, E_{i j}\right]=2 E_{i j} ; j=i+$ $1,\left[D_{i i+1}, E_{i j}\right]=E_{i j} ; j \neq i+1>\bmod \mathcal{M}_{0}(S l(n+1, \mathbb{C}))$. We can see that

$$
S l(n+1, \mathbb{C}) \wedge S l(n+1, \mathbb{C})=<D_{i i+1} \wedge D_{i+1 i+2}, D_{i i+1} \wedge E_{i j}>\bmod \mathcal{M}_{0}(S l(n+1, \mathbb{C})) .
$$

Now for all $w \in \mathcal{M}(S l(n+1, \mathbb{C})) \leq S l(n+1, \mathbb{C}) \wedge S l(n+1, \mathbb{C})$, there exist $\alpha_{i}, \beta_{i j} \in \mathbb{C}$, $i, j=1 \ldots n+1$ and $\tilde{w} \in \mathcal{M}_{0}(S l(n+1, \mathbb{C}))$, such that

$$
w=\sum_{i=1}^{n} \alpha_{i}\left(D_{i i+1} \wedge D_{i+1 i+2}\right)+\sum_{i, j=1}^{n} \beta_{i j}\left(D_{i i+1} \wedge E_{i j}\right)+\tilde{w} .
$$

Since $\tilde{\kappa}(w)=0$, we have $\sum_{i=1}^{n} \alpha_{i}\left[D_{i i+1}, D_{i+1 i+2}\right]+\sum_{i, j=1}^{n} \beta_{i j}\left[D_{i i+1}, E_{i j}\right]=0$. Now if $j=$ $i+1$, then $\sum_{i=1}^{n} \alpha_{i} D_{i i+2}+\sum_{i=1}^{n} 2 \beta_{i} E_{i i+1}=\sum_{i=1}^{n} \alpha_{i}\left(E_{i i}-E_{i+2 i+2}\right)+2 \sum_{i=1}^{n} \beta_{i} E_{i i+1}=0$. So 
for all $i=1 \ldots n, \alpha_{i}=\beta_{i}=0$. If $j \neq i+1$, then $\sum_{i=1}^{n} \alpha_{i} D_{i i+2}+\sum_{i, j=1, i<j}^{n} \beta_{i, j} E_{i j}=0$. So for all $i, j, \alpha_{i}=\beta_{i j}=0$. Hence $w \in \mathcal{M}_{0}(S l(n+1, \mathbb{C}))$ and $\mathcal{M}(S l(n+1, \mathbb{C})) \subseteq \mathcal{M}_{0}(S l(n+1, \mathbb{C}))$. Therefore $\tilde{B}_{0}(S l(n+1, \mathbb{C}))=0$.

Let $L$ be one of the odd-dimension orthogonal Lie algebra $S o(2 n+1, \mathbb{C})$. From [17], $S o(2 n+1, \mathbb{C})$ has the basis $H_{i}, K_{i}^{ \pm}, L_{i j}{ }^{ \pm}, M_{i j}{ }^{ \pm}$such that $D_{i j}=E_{i j}-E_{j i}(1 \leq i \neq$ $j \leq 2 n+1), H_{i}:=\sqrt{-1} D_{2 i-12 i}(i=1, \ldots, n), K_{i}^{ \pm}:=D_{2 i-12 n+1} \pm \sqrt{-1} D_{2 i 2 n+1}(i=$ $1, \ldots, n), L_{i j}^{ \pm}:=\left(D_{2 i-12 j-1}-D_{2 i 2 j}\right) \pm \sqrt{-1}\left(D_{2 i-12 j}+D_{2 i 2 j-1}\right)(1 \leq i<j \leq n)$, $M_{i j}{ }^{ \pm}:=\left(D_{2 i-12 j}-D_{2 i 2 j-1}\right) \pm \sqrt{-1}\left(D_{2 i-12 j-1}+D_{2 i 2 j}\right)(1 \leq i<j \leq n)$. Also we have $\left[H_{i}, K_{i}^{ \pm}\right]=\sqrt{-1} D_{2 n+12 i} \pm D_{2 i-12 n+1},\left[H_{i}, L_{i j}{ }^{ \pm}\right]=-\sqrt{-1} D_{2 i-12 j} \pm D_{2 i-12 j-1}$, $\left[H_{i}, M_{i j}{ }^{ \pm}\right]=-\sqrt{-1} D_{2 i-12 j-1} \pm D_{2 i-12 j},\left[K_{i}{ }^{ \pm}, L_{i j}{ }^{ \pm}\right]=\left[K_{i}^{ \pm}, M_{i j}{ }^{ \pm}\right]=\left[L_{i j}{ }^{ \pm}, M_{i j}{ }^{ \pm}\right]=0$. So in $\bmod \mathcal{M}_{0}(S o(2 n+1, \mathbb{C}))$, can be see that

$$
S o(2 n+1, \mathbb{C})=<H_{i}, K_{i}^{ \pm}, L_{i j}{ }^{ \pm}, M_{i j}{ }^{ \pm} \mid\left[H_{i}, K_{i}{ }^{ \pm}\right],\left[H_{i}, L_{i j}{ }^{ \pm}\right],\left[H_{i}, M_{i j}{ }^{ \pm}\right]>
$$

and in $\bmod \mathcal{M}_{0}(S o(2 n+1, \mathbb{C})), S o(2 n+1, \mathbb{C}) \wedge S o(2 n+1, \mathbb{C})=$

$$
<H_{i} \wedge K_{i}{ }^{ \pm}, H_{i} \wedge L_{i j}{ }^{ \pm}, H_{i} \wedge M_{i j}{ }^{ \pm}>=<D_{2 i-12 i} \wedge D_{2 i 2 n+1}, D_{2 i-12 i} \wedge D_{2 i 2 j}>.
$$

Now for all $w \in \mathcal{M}(S o(2 n+1, \mathbb{C})) \leq S o(2 n+1, \mathbb{C}) \wedge S o(2 n+1, \mathbb{C})$, there exist $\alpha_{1}, \alpha_{2} \in \mathbb{C}$ and $\tilde{w} \in \mathcal{M}_{0}(S o(2 n+1, \mathbb{C}))$, such that $w=\alpha_{1}\left(D_{2 i-12 i} \wedge D_{2 i 2 n+1}\right)+\alpha_{2}\left(D_{2 i-12 i} \wedge D_{2 i 2 j}\right)+\tilde{w}$. Since $\tilde{\kappa}(w)=0$, we have $\alpha_{1}\left[D_{2 i-12 i}, D_{2 i 2 n+1}\right]+\alpha_{2}\left[D_{2 i-12 i}, D_{2 i 2 j}\right]=\alpha_{1} D_{2 i-12 n+1}+\alpha_{2} D_{2 i-12 j}$ $=\alpha_{1}\left(E_{2 i-12 n+1}-E_{2 n+12 i-1}\right)+\alpha_{2}\left(E_{2 i-12 j}-E_{2 j 2 i-1}\right)=0$. Thus $\alpha_{1}=\alpha_{2}=0$. Hence $\mathcal{M}(S o(2 n+1, \mathbb{C})) \subseteq \mathcal{M}_{0}(S o(2 n+1, \mathbb{C}))$, so $\tilde{B}_{0}(S o(2 n+1, \mathbb{C}))=0$.

Let $L$ be an even-dimension orthogonal Lie algebra $S o(2 n, \mathbb{C})$. From [17], $S o(2 n, \mathbb{C})$ has the basis $H_{i}, L_{i j}{ }^{ \pm}, M_{i j}{ }^{ \pm}$, such that $D_{i j}=E_{i j}-E_{j i}(1 \leq i \neq j \leq 2 n+1)$, $H_{i}:=\sqrt{-1} D_{2 i-12 i}(i=1, \ldots, n), L_{i j}^{ \pm}:=\left(D_{2 i-12 j-1}-D_{2 i 2 j}\right) \pm \sqrt{-1}\left(D_{2 i-12 j}+D_{2 i 2 j-1}\right)(1 \leq$ $i<j \leq n), M_{i j}{ }^{ \pm}:=\left(D_{2 i-12 j}-D_{2 i 2 j-1}\right) \pm \sqrt{-1}\left(D_{2 i-12 j-1}+D_{2 i 2 j}\right)(1 \leq i<j \leq n)$. Also we have $\left[H_{i}, L_{i j}^{ \pm}\right]=-\sqrt{-1} D_{2 i-12 j} \pm D_{2 i-12 j-1},\left[H_{i}, M_{i j}^{ \pm}\right]=-\sqrt{-1} D_{2 i-12 j-1} \pm D_{2 i-12 j}$, $\left[L_{i j}^{ \pm}, M_{i j}^{ \pm}\right]=0$. Thus

$$
S o(2 n, \mathbb{C})=<H_{i}, L_{i j}{ }^{ \pm}, M_{i j}{ }^{ \pm} \mid\left[H_{i}, L_{i j}\right],\left[H_{i}, M_{i j}\right]>\bmod \mathcal{M}_{0}(S o(2 n, \mathbb{C})) .
$$

We can write

$$
S o(2 n, \mathbb{C}) \wedge S o(2 n, \mathbb{C})=<H_{i} \wedge L_{i j}{ }^{ \pm}, H_{i} \wedge M_{i j}{ }^{ \pm}>\bmod \mathcal{M}_{0}(S o(2 n, \mathbb{C})) .
$$

Now for all $w \in \mathcal{M}(S o(2 n, \mathbb{C})) \leq S o(2 n, \mathbb{C}) \wedge S o(2 n, \mathbb{C})$, there exist $\alpha_{1}, \alpha_{2} \in \mathbb{C}$ and $\tilde{w} \in \mathcal{M}_{0}(S o(2 n, \mathbb{C}))$, such that $w=\alpha_{1}\left(H_{i} \wedge L_{i j}{ }^{ \pm}\right)+\alpha_{2}\left(H_{i} \wedge M_{i j}{ }^{ \pm}\right)+\tilde{w}$. Since $\tilde{\kappa}(w)=0$, we have $\alpha_{1}\left[H_{i}, L_{i j}{ }^{ \pm}\right]+\alpha_{2}\left[H_{i}, M_{i j}{ }^{ \pm}\right]=\alpha_{1}\left(-\sqrt{-1} D_{2 i-12 j} \pm D_{2 i-12 j-1}\right)+$ $\alpha_{2}\left(-\sqrt{-1} D_{2 i-12 j-1} \pm D_{2 i-12 j}\right)=0$. Thus $\alpha_{1}=\alpha_{2}=0$. Hence

$\mathcal{M}(S o(2 n, \mathbb{C})) \subseteq \mathcal{M}_{0}(S o(2 n, \mathbb{C}))$, so $\tilde{B}_{0}(S o(2 n, \mathbb{C}))=0$.

Let $L$ be a symplectic Lie algebra $S p(n, \mathbb{C})$. From [17], $S p(n, \mathbb{C})$ has the basis $H_{i}, X_{i j}$, $Y_{i j}, Z_{i j}, U_{i}, V_{i}$, such that $H_{i}=E_{i i}-E_{n+i n+i}(1 \leq i \leq n), X_{i j}:=E_{i j}-E_{n+j n+i}(1 \leq$ $i \neq j \leq n), Y_{i j}:=E_{i n+j}+E_{j n+i}(1 \leq i<j \leq n), Z_{i j}:=E_{n+i j}+E_{n+j i}(1 \leq i<$ $j \leq n), U_{i}:=E_{i n+i}(1 \leq i \leq n)$, and $V_{i}:=E_{n+i i}(1 \leq i \leq n)$. Since $\left[X_{i j}, Y_{i j}\right]=$ $2 E_{i n+i},\left[X_{i j}, Z_{i j}\right]=-2 E_{n+j j},\left[X_{i j}, V_{i}\right]=-E_{n+i j}-E_{n+j i},\left[Y_{i j}, Z_{i j}\right]=E_{i i}+E_{j j},\left[Y_{i j}, V_{i}\right]=$ $-E_{n+i n+j}+E_{j i},\left[Z_{i j}, U_{i}\right]=-E_{i j}+E_{n+j n+i},\left[U_{i}, V_{i}\right]=E_{i i},\left[X_{i j}, U_{i}\right]=\left[Y_{i j}, U_{i}\right]=\left[Z_{i j}, V_{i}\right]=$ $0,\left[H_{i}, X_{i j}\right]=-E_{n+j n+i},\left[H_{i}, Y_{i j}\right]=E_{i n+j}-E_{j n+i},\left[H_{i}, Z_{i j}\right]=-E_{n+i j}-E_{n+j i},\left[H_{i}, U_{i}\right]=$ $2 E_{i n+i}$ and $\left[H_{i}, V_{i}\right]=-2 E_{n+i i}$, we have

$S p(n, \mathbb{C})=<H_{i}, X_{i j}, Y_{i j}, Z_{i j}, U_{i}, V_{i} \mid\left[X_{i j}, Y_{i j}\right],\left[X_{i j}, Z_{i j}\right],\left[X_{i j}, V_{i}\right],\left[Y_{i j}, Z_{i j}\right],\left[Y_{i j}, V_{i}\right],\left[Z_{i j}, U_{i}\right]$ , $\left[U_{i}, V_{i}\right],\left[H_{i}, X_{i j}\right],\left[H_{i}, Y_{i j}\right],\left[H_{i}, Z_{i j}\right],\left[H_{i}, U_{i}\right],\left[H_{i}, V_{i}\right]>\bmod \mathcal{M}_{0}(S p(n, \mathbb{C}))$. 
We can write

$$
\begin{gathered}
S p(n, \mathbb{C}) \wedge S p(n, \mathbb{C})=<X_{i j} \wedge Y_{i j}, X_{i j} \wedge Z_{i j}, X_{i j} \wedge V_{i}, Y_{i j} \wedge Z_{i j}, Y_{i j} \wedge V_{i}, Z_{i j} \wedge U_{i}, \\
U_{i} \wedge V_{i}, H_{i} \wedge X_{i j}, H_{i} \wedge Y_{i j}, H_{i} \wedge Z_{i j}, H_{i} \wedge U_{i}, H_{i} \wedge V_{i}>\bmod \mathcal{M}_{0}(S p(n, \mathbb{C})) .
\end{gathered}
$$

By using a similar method, the result follows.

Since $S o(2, \mathbb{C})$ is commutative, its Bogomolov multiplier is trivial. Therefore, we have

Corollary 7.5. Following complex Lie algebras, have trivial Bogomolov multipliers.

(i) $\operatorname{Sl}(n+1, \mathbb{C})$,

(ii) $\operatorname{So}(2 n+1, \mathbb{C})$,

(iii) $S p(n, \mathbb{C})$,

(iv) $S o(2 n, \mathbb{C})$.

Complex simple Lie algebras have been completely classified by Cartan [5]. They are classified into four infinite classes with five exceptional Lie algebras.

Theorem 7.6 ([10]). Every simple Lie algebra over $\mathbb{C}$ is isomorphic to precisely one of the following Lie algebras

(i) $\operatorname{Sl}(n+1, \mathbb{C}), n \geq 1$,

(ii) $S o(2 n+1, \mathbb{C}), n \geq 2$,

(iii) $\operatorname{Sp}(n, \mathbb{C}), n \geq 3$,

(iv) $S o(2 n, \mathbb{C}), n \geq 4$,

(v) The exceptional Lie algebras $G_{2}, F_{4}, E_{6}, E_{7}$ and $E_{8}$.

Knapp in [17] showed that the five exceptional Lie algebras $G_{2}, F_{4}, E_{6}, E_{7}, E_{8}$ have dimensions $14,52,78,133$ and 248 , respectively.

Theorem 7.7. Every complex simple Lie algebra has a trivial Bogomolov multiplier.

Proof. By using Theorems 7.4 and 7.6, all complex simple Lie algebras in (i), (ii), (iii) and (iv) have trivial Bogomolov multipliers. Also we know any simple Lie algebra is semisimple. Thus the Example 2 of Chapter 2 in [2] showed that the exceptional Lie algebras $G_{2}, F_{4}, E_{6}, E_{7}$ and $E_{8}$ have trivial Bogomolov multipliers.

\section{References}

[1] A. Bak, G. Donadze, N. Inassaridze and M. Ladra, Homology of multiplicatie Lie ring, J. Pure Appl. Algebra 208, 761-777, 2007.

[2] P.G. Batten, Multipliers and covers of Lie algebras, ph. D. diss, North carolina state university, 1993.

[3] P.G. Batten, K. Moneyhun and E. Stitzinger, On characterizing nilpotent Lie algebras by their multipliers, Comm. Algebra 24 (14), 4319-4330, 1996.

[4] F.A. Bogomolov, The Brauer group of quotient spaces of linear representations, (Russian) Izv. Akad. Nauk SSSR Ser. Mat. 51 (3), 485-516, 688; translation in Math. USSR-Izv. 30 (3), 455-485, 1987.

[5] E. Cartan, Sur la Reduction a sa Forme Canonique de la Structure d'un Groupe de Transformations Fini et Continu, (French) Amer. J. Math. 18 (1) 1-61, 1896.

[6] Y. Chen and R. Ma, Some groups of order $p^{6}$ with trivial Bogomolov multipliers, arxiv: $1302.0584 \mathrm{v} 5$.

[7] S. Cicalo, W.A. de Graaf and C. Schneider, Six-dimensional nilpotent Lie algebras, Linear Algebra Appl. 436 (1), 163-189, 2012.

[8] G. Ellis, Nonabelian exterior products of Lie algebras and an exact sequence in the homology of Lie algebras, J. Pure Appl. Algebra 46 (2-3), 111-115, 1987.

[9] W.A. de Graaf, Classification of 6-dimensional nilpotent Lie algebras over fields of characteristic not 2, J. Algebra 309 (2), 640-653, 2007. 
[10] B.C. Hall, Lie groups, Lie algebras and representations, An elementary introduction. Graduate Texts in Mathematics, 222, Springer-Verlag, New York, xiv+351 pp, 2003.

[11] P. Hardy, On characterizing nilpotent Lie algebras by their multipliers. III, Comm. Algebra 33 (11), 4205-4210, 2005.

[12] P. Hardy and E. Stitzinger, On characterizing nilpotent Lie algebras by their multipliers $t(L)=3$, 4, 5, 6, Comm. Algebra 26 (11), 3527-3539, 1998.

[13] U. Jezernik and P. Moravec, Universal commutator relations, Bogomolov multipliers and commuting probability, J. Algebra 428, 1-25, 2015.

[14] U. Jezernik and P. Moravec, Commutativity preserving extensions of groups, Proc. Roy. Soc. Edinburgh Sect. A 148 (3), 575-592, 2018.

[15] M.R. Jones, Multiplicators of p-groups, Math. Z. 127, 165-166, 1972.

[16] M. Kang, Bogomolov multipliers and retract rationality for semidirect products, J. Algebra 397, 407-425, 2014.

[17] A.W. Knapp, Lie groups beyond an introduction, Second edition. Progress in Mathematics, 140. Birkhäuser Boston, Inc., Boston, MA, xviii+812 pp, 2002.

[18] B. Kunyavskii, The Bogomolov multiplier of finite simple groups, Cohomological and geometric approaches to rationality problems, 209-217, Progr. Math, 282, Birkhauser Boston, Inc, Boston, MA, 2010.

[19] M. Lazard, Sur les groupes nilpotents et les anneaux de Lie, (French) Ann. Sci. Ecole Norm. Sup. 71 (3), 101-190, 1954.

[20] S. Lie, Theorie der Transformationsgruppen I, (German) Math. Ann. 16 (4), 441-528, 1880.

[21] K. Moneyhun, Isoclinisms in Lie algebras, Algebras Groups Geom, (English summary) Algebras Groups Geom. 11 (1), 9-22, 1994.

[22] P. Moravec, Unramified Brauer groups of finite and infinite groups, Amer. J. Math. 134 (6), 1679-1704, 2012.

[23] P. Niroomand, On dimension of the Schur multiplier of nilpotent Lie algebras, Cent. Eur. J. Math. 9 (1), 57-64, 2011.

[24] P. Niroomand and M. Parvizi, 2-Nilpotent multipliers of a direct product of Lie algebras, Rend. Circ. Mat. Palermo (2) 65 (3), 519-523, 2016.

[25] D.J. Saltman, Noether's problem over an algebraically closed field, Invent. Math. 77 (1), 71-84, 1984.

[26] H. Samelson, Notes on Lie algebras, Van Nostrand Reinhold Mathematical Studies, No. 23 Van Nostrand Reinhold Co., New York- London-Melbourne, vi+165 pp, (loose errata), 1969.

[27] V.S. Varadarajan, Lie groups, Lie algebras and their representations, Reprint of the 1974 edition. Graduate Texts in Mathematics, 102, Springer-Verlag, New York, 1984.

[28] J.B. Zuber, Invariances in physics and group theory, Sophus Lie and Felix Klein: the Erlangen program and its impact in mathematics and physics, 307-326, IRMA Lect. Math. Theor. Phys. 23, Eur. Math. Soc. Zürich, 2015. 